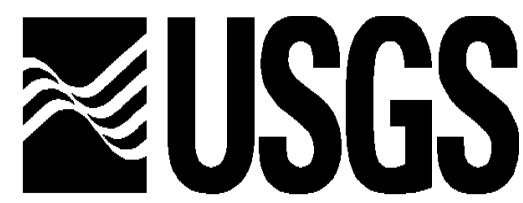

science for a changing world

\title{
Manual del McVCO 1999
}

porP. J. MCCHESNEY ${ }^{1}$

\section{OPEN-FILE REPORT 99-361}

\section{9}

ESTE INFORME ES PRELIMINAR Y NO HA SIDO REVISADO PARA SU CONFORMIDAD SEGÚN LAS NORMAS DE LA EDITORIAL DEL SERVICIO GEOLÓGICO DE LOS ESTADOS UNIDOS (U.S. GEOLOGICAL SURVEY). CUALQUIER USO DE LOS NOMBRES DEL PRODUCTO, LA FIRMA O LA EMPRESA, ES SOLAMENTE CON PROPÓSITOS DESCRIPTIVOS Y NO IMPLICA NINGÚN TIPO DE APOYO POR EL GOBIERNO DE LOS ESTADOS UNIDOS. AUNQUE ESTE DISPOSITIVO Y EL PROGRAMA QUE CONTIENE HAN SIDO UTILIZADOS POR EL USGS, ÉSTE NO DA NINGUNA GARANTÍA, EXPRESA O IMPLÍCITA, CON RESPECTO A LA EXACTITUD Y EL FUNCIONAMIENTO DEL MISMO. TAMPOCO CONSTITUYE NINGUNA GARANTÍA EL HECHO DE SU DISTRIBUCIÓN, Y EL USGS NO ASUME NINGUNA RESPONSABILIDAD CON RESPECTO A LO YA MENCIONADO.

Department of the Interior U.S. Geological Survey

${ }^{1}$ University of Washington Geophysics Program

USGS Cascades Volcano Observatory

5400 MacArthur Blvd.

Vancouver WA 98661

\section{University of Washington} Geophysics Program 


\title{
U.S. Department of the Interior BRUCE BABBIT, SECRETARY
}

\author{
U.S. Geological Survey \\ CHARLES GROAT, DIRECTOR
}

Este informe sólo está disponible en forma digital en la Red Mundial. URL: http://geopubs.wr.usgs.gov/open-file/of99-361s/ 


\section{Manual del McVCO 1999: Introducción al version en Español}

El Manuel del McVCO1999 fue traducido por:

Armando Gutierrez, Portland, Oregon, EEUU

$\mathrm{y}$

Dr. Roberto Quaas, Centro Nacional de Prevención de Desastres (CENAPRED), Mexico con contribuciónes por

Andrew Lockhart, U. S. Geological Survey, Vancouver, Washington, EEUU. 


\section{Contenido}

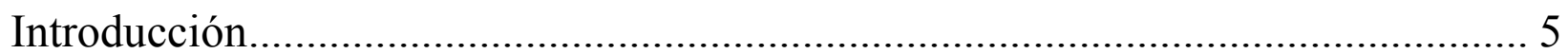

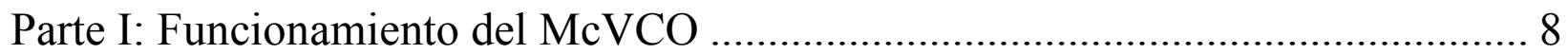

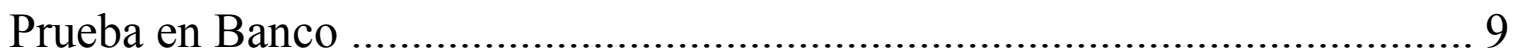

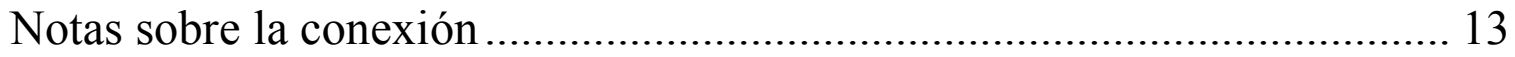

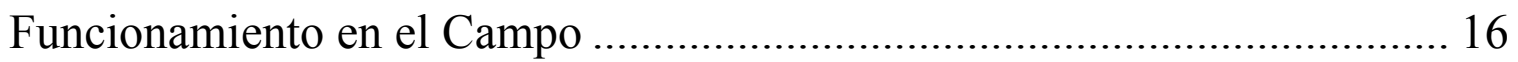

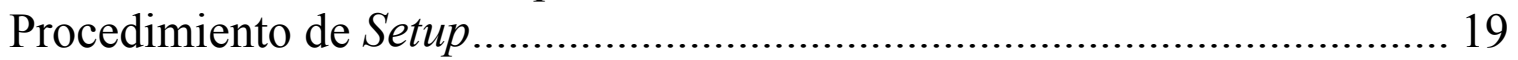

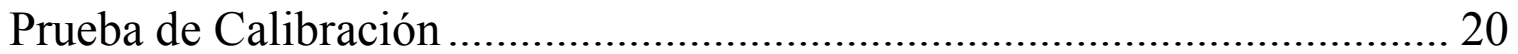

Figura 2A. Ubicación de las Partes e Interruptor DIP ................................ 22

Figura 2B. Pins de Salida y Entrada y Puntos de Prueba............................. 23

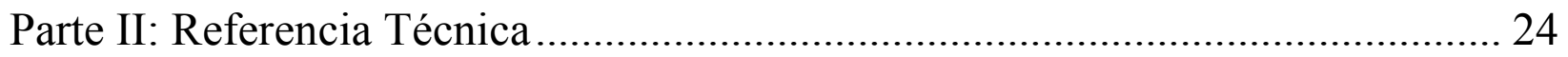

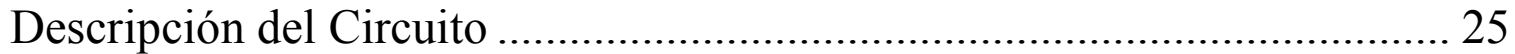

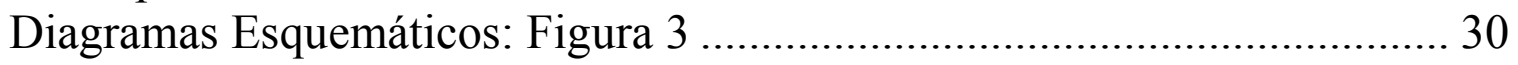

Especificaciones ................................................................................... 35

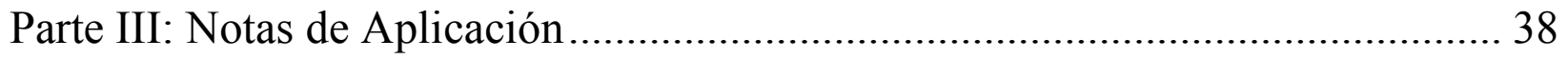

Respuesta en Frecuencia y Ganancia......................................................... 39

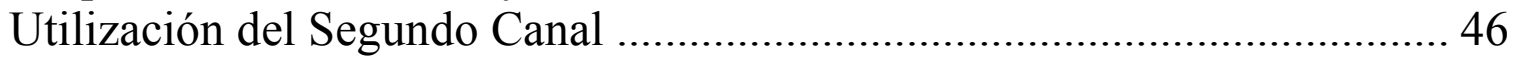

Ciclo de Prueba del McVCO ...................................................................... 49

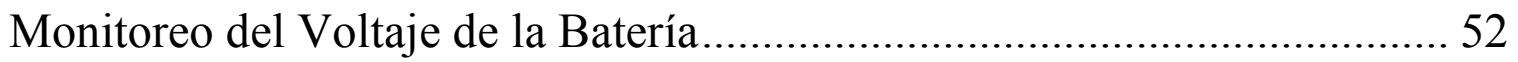

Utilización de la Salida de Potencia Conmutada ......................................... 54

Conexión de Tres Componentes ................................................................... 56 


\section{Introducción}

El McVCO es un generador de frecuencias basado en un microcontrolador que reemplaza al oscilador controlado por voltaje (VCO) utilizado en telemetría analógica de datos sísmicas. Acepta señales de baja potencia desde un sismómetro y produce una señal subportadora modulada en frecuencia adecuada para enlaces telefónicos o vía radio a un lugar remoto de recolección de datos. La frecuencia de la subportadora y la ganancia pueden ser seleccionadas mediante un interruptor. Tiene la opción de poder operar con dos canales para la observación con ganancia alta y baja. La Figura 1 muestra el diagrama de bloques del instrumento.

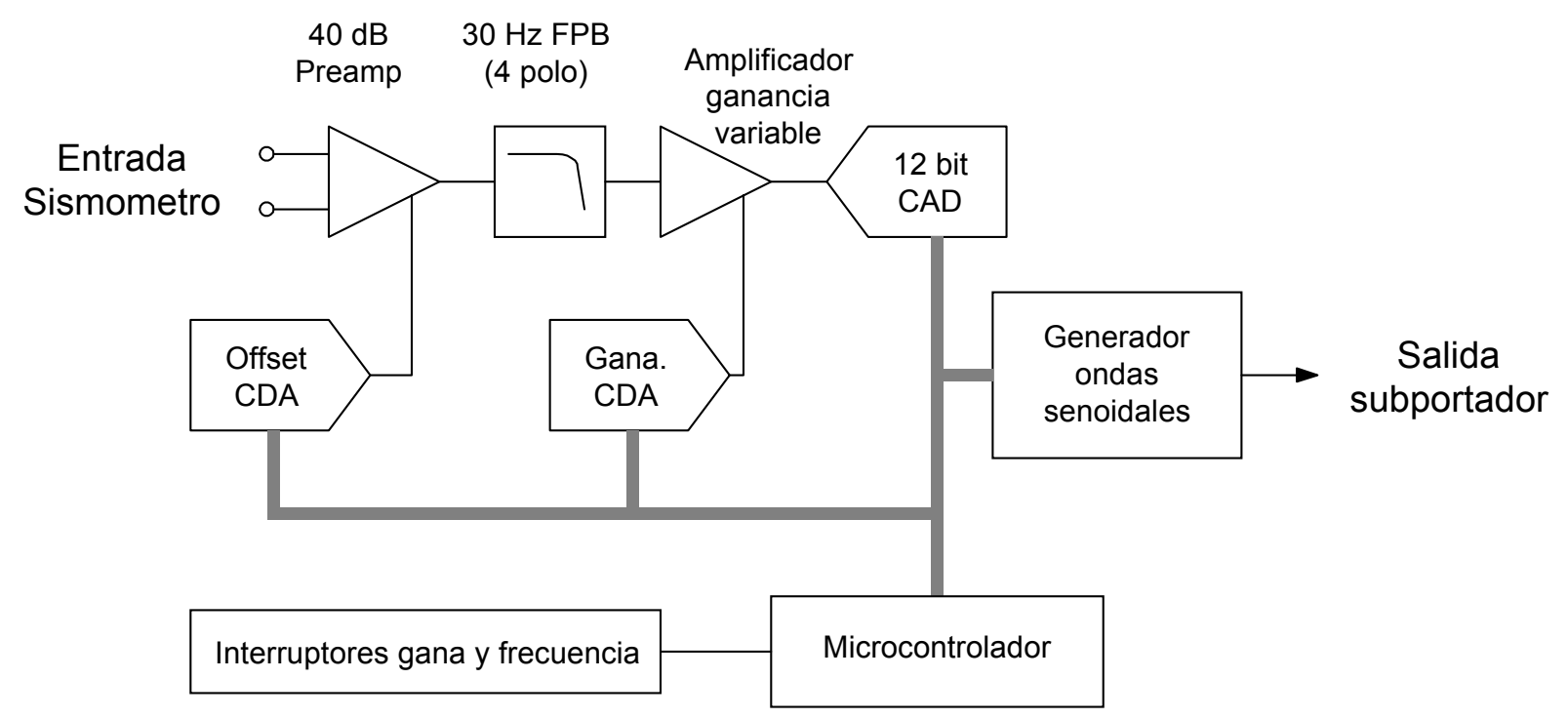

Figura 1. Diagrama de bloques del Oscilador Controlado por Voltaje basado en un Microcontrolador (McVCO).

A diferencia del $\mathrm{VCO}$ hecho con circuitos analógicos, el McVCO utiliza la conversión analógica a digital (CAD) para muestrear la señal sísmica a la salida del amplificador. El valor de cada muestra determina el código enviado al generador programable de ondas senoidales. El generador sintetiza la frecuencia senoidal requerida para que sea proporcional a la de la muestra. El ciclo de muestreo y sintetización se repite varios cientos de veces por segundo para producir una señal de audio que es modulada en frecuencia por la señal sísmica. La ventaja del método del $\mathrm{McVCO}$ de producir una señal modulada, es que no depende de las variaciones por envejecimiento de los componentes ni de efectos de temperatura que reducen la estabilidad de los VCOs analógicos.

Además de calcular la modulación, el microcontrolador ejecuta una subrutina de reducción del offset que incrementa la estabilidad del McVCO. Los offsets creados por los componentes analógicos del amplificador de la señal sísmica son restados por el offset del conversor digital- 
analógico (CDA) en el amplificador de entrada. La supresión del offset ayuda a la operación en la frecuencia central deseada. Otro CDA brinda el control de ganancia del microcontrolador. Esto permite que el instrumento se adapte a varios patrones de sensibilidad de modulación por medio de tablas de ganancia.

El McVCO fue diseñado con el propósito de mejorar la telemetría analógica de las señales dentro de la Pacific Northwest Seismograph Network (PNSN) (Red Sismográfica del Noroeste del Pacífico). Su desarrollo recibió el respaldo del Programa de Geofísica de la Universidad de Washington y del "Volcano Hazards and Earthquake Hazards programs of the United States Geological Survey (USGS) (Programa de Investigaciones de Riesgos Volcánicos y Programa de Investigaciones de Riesgos Sísmicos de los EEUU). Cientos de instrumentos se han construido e instalado. Además de utilizarlo el PNSN, el McVCO es usado por el Observatorio Vulcanológico de Alaska para monitorear los volcanes aleutianos y por el USGS Volcano Disaster Assistance Program (Programa de Ayuda en las Catástrofes Volcánicas del USGS) para responder a crisis volcánicas en otros países.

La Parte I de este manual cubre el funcionamiento del McVCO. Trata de dar al usuario la suficiente información para conectar la unidad, revisar su funcionamiento e instalarla en el campo. El McVCO posee algunas características diferentes al VCO analógico. Por consiguiente, se recomienda ver la sección de la Prueba de Banco para los nuevos usuarios, ya que demuestra las cualidades del funcionamiento y control únicas del McVCO. Las Figuras $2 \boldsymbol{A}$ y $2 \boldsymbol{B}$ son resúmenes de la información requerida para operar correctamente el McVCO.

La Parte II del manual es una referencia técnica para aquellos que necesitan saber con más detalle cómo funciona el McVCO. Un técnico experimentado encontrará suficientes detalles para diagnosticar y reparar la mayoría de las fallas.

La Parte III cubre una serie de temas que requieren un trato explícito o que derivan del despliegue del instrumento. Se espera que estas notas de aplicación sean ampliadas y los usuarios del McVCO motivados a enviar sus preguntas y experiencias. 
La intención de este manual es proveer la información necesaria para entender y operar el McVCO. Aquellos que deseen construir sus propios instrumentos basados en la información presentada aquí, pueden hacerlo. Sin embargo, el autor niega expresamente las garantías implícitas de comercialización y la adaptación para cualquier propósito determinado. Es responsabilidad del fabricante del instrumento probar y verificar el funcionamiento del mismo. Las especificaciones dadas en este manual son solamente para guía y no son una garantía de su funcionamiento.

El código del microcontrolador requerido para construir el McVCO no está incluido en este manual. Aquellos que deseen comprar microcontroladores programables, o intercambiar opiniones sobre el McVCO pueden contactarse con:

\author{
P. J. McChesney \\ Stone Code \\ $305{\mathrm{E} 24^{\text {th }}}$ Street \\ Vancouver, WA 98663 \\ USA
}


Parte I. Funcionamiento del McVCO

\section{Parte I: Funcionamiento del McVCO}




\section{Prueba en Banco}

Para realizar la prueba en banco del $\mathrm{McVCO}$ se requiere lo siguiente:

A) Una fuente de alimentación o una batería de 12 VCD.

B) Un contador de frecuencia de gama audible o un multímetro digital de lectura de frecuencias con resolución de $1 \mathrm{~Hz}$.

C) Batería de $1.5 \mathrm{VCD}$, resistencias de $100 \Omega$ y $100 \mathrm{~K} \Omega$. Utilice resistencias del $1 \%$ para la calibración.

\section{NO SUMINISTRE ENERGIA HASTA QUE SE LE INDIQUE.}

Examine la Figura $2 A$ al final de la Parte I. La sección superior tiene un gráfico que muestra la ubicación de los componentes principales del McVCO. Nótese en particular la ubicación del interruptor DIP en la esquina superior derecha de la tarjeta y los bornes de reset en el borde inferior de la misma hacia la derecha.

\section{1) Ajuste del Interruptor DIP}

El interruptor DIP controla la frecuencia de la subportadora y la ganancia. El interruptor superior es el interruptor 1 y el inferior, el 9. Los interruptores 1, 2 y 3 controlan la ganancia del canal de ganancia variable. Los interruptores 4, 5 y 6 controlan la frecuencia de la subportadora del canal de ganancia variable f1. Cuando la tarjeta está equipado para el funcionamiento de dos canales, los interruptores 7, 8 y 9 controlan la frecuencia de la subportadora de baja ganancia fija $\mathrm{f} 2$. La tarjeta del circuito impreso del McVCO está marcado para mostrar las funciones del interruptor.

En la parte inferior de la Figura $2 A$ se muestran las posiciones del interruptor DIP para la selección de frecuencias y ganancias. Un interruptor puesto en 0 indica que éste está cerrado y que la palanca del mismo está colocada hacia el borde de la tarjeta. Un interruptor puesto en 1 está abierto y su palanca colocada hacia el centro de la tarjeta.

Ejemplo:

Ajuste los interruptores para que la ganancia sea de $60 \mathrm{~dB}, \mathrm{fl}=680 \mathrm{~Hz}$ y f2 $=3060 \mathrm{~Hz}$

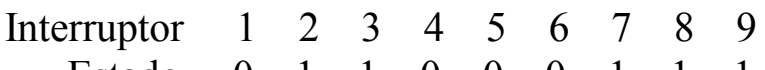

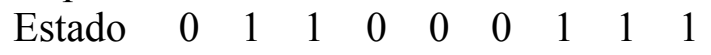

Para la alimentación de la tarjeta se conecta el positivo de la fuente o la batería al punto $\mathbf{B}+$ y el negativo a TIERRA (GND). Los puntos B+ y GND se encuentran en la esquina izquierda inferior de la tarjeta. Si la tarjeta está embalada, consulte los diagramas de conexión y alambrado para una correcta instalación.

Conecte un contador de frecuencia entre TP5 y GND y ENCIENDA LA FUENTE DE ALIMENTACION. El contador muestra $680 \mathrm{~Hz}$ durante 10 segundos mientras el instrumento se 
Parte I. Funcionamiento del McVCO

ajusta a cero automáticamente. Luego de 10 segundos la unidad comienza una rutina de prueba que dura 42 segundos y el conteo variará por encima y por debajo de $680 \mathrm{~Hz}$. Después de la rutina de prueba la salida se fijará en $680 \mathrm{~Hz} \pm 2 \mathrm{~Hz}$. Las versiones anteriores del programa no ejecutan la rutina de prueba de 42 segundos y algunas versiones posteriores tienen períodos de prueba más largos.

\section{2) $\underline{\text { Reset }}$}

Cambie el interruptor DIP a Sw4=0, Sw5=0, Sw6=1. Nótese que no hay ningún cambio en la frecuencia. No sucede nada cuando los interruptores DIP son cambiados hasta que la unidad es restaurada a cero (reset) otra vez. Busque los bornes de reset en el borde inferior derecho de la tarjeta. Toque ambos pins al mismo tiempo con un objeto conductor como una punta de prueba o la punta de un desarmador. La unidad se restaura a cero cuando ya no existe cortocircuito entre los pins.

De un reset a la unidad. Ahora la frecuencia cambia a $1020 \mathrm{~Hz}$ y luego de los 10 seg del ajuste automático a cero de la rutina de prueba de $42 \mathrm{seg}$, el conteo se fijará alrededor de $2 \mathrm{~Hz}$ de 1020 $\mathrm{Hz}$.

\section{3) Cambio de Frecuencia}

Las posiciones del interruptor para las otras frecuencias fl se encuentran en el cuadro central de la Figura 2A. Trabaje con ellos. A menos que seleccione $3060 \mathrm{~Hz}$, la frecuencia cambiará cada vez que la unidad sea restaurada a cero.

Cuando seleccione $3060 \mathrm{~Hz}$ para f1, la unidad pasará a un modo especial de suspención y el contador mostrará $0 \mathrm{~Hz}$. Observe los interruptores 7, 8 y 9 y compárelos con 4, 5 y 6 . Están iguales, y el interruptor está puesto de modo que $\mathrm{f} 1=\mathrm{f} 2$. Cada vez que Sw4 = Sw7 y Sw5 = Sw8 y Sw6 = Sw9, la unidad se colocará en un modo especial de suspención utilizado para realizar algunos ajustes (ver Procedimiento de Setup). Esto sucede incluso si f2 no es utilizado. Si la unidad no produce ninguna salida, observe si el interruptor DIP está ajustado para que f1 = f y en el modo de suspención.

Cambie cualquier interruptor $\mathrm{f} 2(7,8,9)$. De un reset a la unidad y asegúrese de que la misma no se encuentre apagada y que el contador de frecuencia muestre $3060 \mathrm{~Hz} \pm 2 \mathrm{~Hz}$.

El segundo canal se activará si el chip generador de f2, U16, se encuentra instalado y el pin 16 del microcontrolador, U9, está conectado a +5 VCD (ver Figura $2 A$ para su localización). Si es así, ejercite las posiciones del interruptor de f2 luego de mover el contador a TP6. Los interruptores de $\mathrm{f} 2$ trabajan del mismo modo que los interruptores de f1. 


\section{4) Cambio de Ganancia}

Prepare una fuente de señales de aproximadamente $1.5 \mathrm{mV}$ conectando las resistencias de 100 $\mathrm{K} \Omega$ y $100 \Omega$ en series a través de la batería de $1.5 \mathrm{~V}$. Conecte el lado positivo de la batería a un lado de la resistencia de $100 \mathrm{~K} \Omega$. Conecte el lado negativo de la batería a un lado de la resistencia de $100 \Omega$. Una los extremos libres de ambas resistencias. La fuente de señales de $1.5 \mathrm{mV}$ es el voltaje que pasa a través de la resistencia de $100 \Omega$.

Ajuste de los interruptores DIP a G $=60 \mathrm{~dB}, \mathrm{fl}=680 \mathrm{~Hz}$ y f2 $=3060 \mathrm{~Hz}$

\begin{tabular}{|l|l|l|l|l|l|l|l|l|l|}
\hline Interruptor & 1 & 2 & 3 & 4 & 5 & 6 & 7 & 8 & 9 \\
\hline & 0 & 1 & 1 & 0 & 0 & 0 & 1 & 1 & 1 \\
\hline
\end{tabular}

Conecte el contador de frecuencia a TP5 y de un reset a la unidad. Espere alrededor de un minuto para que el ciclo de prueba y el ajuste automático a cero terminen, luego registre la frecuencia fl. La misma será $680 \mathrm{~Hz} \pm 2 \mathrm{~Hz}$.

Conecte la fuente de $1.5 \mathrm{mV}$ con el lado negativo de la batería a I- y la conexión entre las resistencias a $\mathbf{I}+$.

La frecuencia aumentará a un valor entre 720 y $760 \mathrm{~Hz}$. Dicho aumento depende del voltaje exacta de la batería y la calibración utilizada por su sistema. Note el cambio en la frecuencia.

Es necesario desconectar la fuente de $1.5 \mathrm{mV}$ cada vez que se de un reset a la unidad ya que cada reset produce un ajuste automático a cero. Si la fuente de $1.5 \mathrm{mV}$ es conectada durante el ajuste automático a cero, el amplificador tratará de anular la entrada de $1.5 \mathrm{mV}$.

Desconecte la fuente de $1.5 \mathrm{mV}$ y ponga la ganancia en $54 \mathrm{~dB}, \mathrm{Sw} 1=0, \mathrm{Sw} 2=1, \mathrm{Sw} 3=0$.

De un reset a la unidad. Espere más o menos un minuto y reconecte la fuente de $1.5 \mathrm{mV}$ exactamente como estaba antes. Observe que el cambio en la frecuencia es la mitad de lo que era. Conforme se reduce la ganancia a $48 \mathrm{~dB}$, el cambio de frecuencia se reducirá nuevamente a la mitad, mostrando que cada disminución de ganancia de $6 \mathrm{~dB}$, resulta en un cambio de frecuencia de la mitad.

Desconecte la fuente de $1.5 \mathrm{mV}$ y ajuste la ganancia en $84 \mathrm{~dB}: \mathrm{Sw} 1=1, \mathrm{Sw} 2=1, \mathrm{Sw} 3=1$. De un reset a la unidad y espere un minuto antes de reconectar la fuente de $1.5 \mathrm{mV}$. La frecuencia cambiará alrededor de $800 \mathrm{~Hz}$. No importa lo saturado que esté, el McVCO no cambiará la frecuencia de la subportadora más de $125 \mathrm{~Hz}$ de la frecuencia central. 


\section{5) Ajuste automático a cero y compensación del offset}

Para observar la acción del circuito de ajuste automático a cero, ajuste la ganancia a $60 \mathrm{~dB}$ : Sw1 = $0, \mathrm{Sw} 2=1, \mathrm{Sw} 3=1$, y con la fuente de $1.5 \mathrm{mV}$ conectada, de un reset a la unidad. La frecuencia de f1 en TP5 moverá hacia la frecuencia central del canal, $680 \mathrm{~Hz} \pm 1 \mathrm{~Hz}$ durante 10 seg mientras se lleva a cabo el ajuste automático a cero. Al final del ajuste y la rutina de prueba, la frecuencia se establecerá en algún valor mayor que el de la frecuencia central correcta. La entrada de $1.5 \mathrm{mV}$ es demasiado grande para que el circuito de corrección de offset se ponga en cero.

Desconecte la fuente de $1.5 \mathrm{mV}$. La frecuencia disminuirá por debajo de la frecuencia central. Esa es la desviación que se origina debido a la anulación errónea durante el ajuste automática a cero. A veces ocurren anulaciones erróneas en el campo, vea la discusión sobre este tema en la sección de Operación de Campo, Parte I.

La anulación errónea puede corregirse de dos formas. Primero, la unidad activa un sistema de anulación de offset en segundo plano durante la operación normal. La acción de este sistema es muy gradual, ya que su función principal es de cancelar los offsets producidos por los cambios de temperatura y el envejecimiento de los componentes del circuito. Los detalles del sistema de corrección de offset se encuentran en la sección de respuesta en frecuencia, en la Parte III, Respuesta de Frecuencia y Ganancia del McVCO.

Para observar la compensación de offset en segundo plano, provoque una anulación errónea dando un reset a la unidad mientras está conectada a la fuente de $1.5 \mathrm{mV}$. Quite la entrada de $1.5 \mathrm{mV} \mathrm{y}$ espere. Se necesitan un poco más de 18 horas para regresar a la frecuencia central desde un offset extremo. Deje la unidad toda la noche o vaya a almorzar. Cuando regrese, vea cuánto se ha movido la frecuencia hacia a la frecuencia central.

La segunda y más rápida manera para deshacerse de una anulación errónea es dando un reset a la unidad sin que esté conectada a la fuente de $1.5 \mathrm{mV}$ y dejando que sola vuelva a cero. No trate de anular la unidad con P1, el potenciómetro de anulación, sin pasar por el Procedimiento de Setup, que se encuentra descrito en la Parte I.

Ya ha visto el funcionamiento básico del McVCO. Como se muestra en la Figura 2A, el interruptor DIP selecciona la ganancia y la frecuencia. El reset hace que los interruptores sean leídos y comience un ajuste automático a cero y un ciclo de prueba que dura alrededor de un minuto antes que el McVCO comience a funcionar normalmente. Durante la operación el offset es corregido por un esquema de compensación a largo plazo. Los ciclos de prueba difieren según las versiones del McVCO. Vea Ciclo de Prueba del McVCO en la Parte III para su descripción. 
Parte I. Funcionamiento del McVCO

\section{Notas sobre la conexión}

El McVCO no está provisto de un embalaje estándar y la mayoría de los usuarios proveen el gabinete, los conectores y la protección adecuados contra la intemperie según las circunstancias.

El diseño del McVCO lo hace relativamente insensible a interferencias eléctricas y la principal consideración de su embalaje es para protegerlo de la humedad y el esfuerzo mecánico. El grado de protección requerido se deja a juicio del usuario. Lo que sigue son algunas sugerencias sobre la conexión eléctrica y la descripción de algunas opciones de conexión de los circuitos. Ver Figura $2 A$ para las ubicaciones del montaje.

\section{Conexión del Sismómetro}

El McVCO debe tener una entrada de dos conductores con ambos lados aislados de la tierra física. Ambos conductores deben estar dentro de un cable blindado. El conductor conectado al lado positivo de la bobina del sismómetro se conecta a I+ en el McVCO. El conductor conectado al lado negativo de la bobina se conecta a I-. Si fuera necesario, existe un punto de soldadura (no marcado) conectado a tierra del $\mathrm{McVCO}$ entre I+ e I- para el blindaje del cable del sismómetro.

No permita que el blindaje del sismómetro se transforme en un conductor de corriente. Asegúrese que esté conectado a tierra en un sitio solamente. Si el blindaje está conectado a tierra en la cubierta de un conector, no lo conecte a tierra en I+ e I-. El blindaje debe prolongarse cerca de I+ e I- incluso si no está conectado a tierra allí.

\section{Alimentación Eléctrica y Salida}

Conexiones de la alimentación

Las conexiones recomendadas para la alimentación eléctrica son en los puntos de soldadura $\mathbf{B}+\mathrm{y}$ GND con el conductor de alimentación positivo conectado a $\mathbf{B}+$. El punto de soldadura $\mathbf{B}+$ está protegido contra la inversión de polaridad por un diodo en serie. Para las tarjetas marcadas MCFM05, B+ ubicado en los bornes de J1 y J2 está en el lado opuesto del diodo de protección del punto de soldadura $\mathbf{B}+$. Las tarjetas más nuevas, marcadas $\mathrm{McVCO}-01$, tienen el punto de soldadura B+ y el B- en J1 y J2 conectados directamente.

$\mathbf{S W}+$ es una salida de potencia conmutada. Provee el voltaje de entrada $\mathbf{B}+$ menos aproximadamente $0.5 \mathrm{VCD}$ a $200 \mathrm{~mA}$. SW+ se apaga cuando la fuente de alimentación de entrada cae aproximadamente por debajo de $10.5 \mathrm{VCD}$ y no se enciende hasta que la energía vuelve a más o menos 11.5 VCD.

SW+ debe usarse con cuidado. Si hay suficiente resistencia en las conexiones entre el McVCO y la fuente de alimentación, el circuito oscilará. Esto ocurre cuando la caída de voltaje a través de la conexión resistiva a la fuente de alimentación es mayor que la diferencia de 1 volt entre los niveles de encendido y apagado. Por ejemplo, si un transmisor alimentado desde $\mathbf{S W}+$ toma 80 $\mathrm{mA}$, esta corriente, mas los $20 \mathrm{~mA}$ de corriente del McVCO se combinan para una corriente total 
Parte I. Funcionamiento del McVCO

de $100 \mathrm{~mA}$. La resistencia entre el McVCO y la fuente de alimentación debe mantenerse en menos de $10 \Omega$, $(\mathrm{R}=1 \mathrm{VDC} / 0.1 \mathrm{~A})$. Para un margen de seguridad adecuado, la resistencia de la conexión debe ser menor de $5 \Omega$ con una carga total de $100 \mathrm{~mA}$. Las conexiones en la mayoría de las instalaciones son mucho menores que ésta, pero la corrosión puede aumentar la resistencia total y causar oscilación.

No utilice $\mathbf{S W}+$ cuando celdas de aire (Air Cell batteries) u otras fuentes de alimentación resistivas son utilizadas como fuentes de alimentación. El circuito oscilará mientras la caída de voltaje interna rebase los niveles ON y OFF del circuito. Ver la Parte III, "Utilización de Salida de Potencia Conmutada, SW+" para una descripción más detallada.

Salidas de la subportadora

La subportadora de audiofrecuencia está provista en Car+ y Car-. Ambas conexiones quedan aisladas con respecto a tierra. Si se desea una salida de referencia a tierra, Car- puede ser conectada a tierra fuera de la tarjeta o en los puntos de soldadura ubicados cerca de la esquina inferior izquierda del transformador de salida $\mathrm{T} 1$.

Car+ está acoplado de forma capacitiva al transformador de salida para proveer aislamiento de CD. Como en cualquier salida acoplada capacitivamente, esto puede causar un recorte no deseado con algunas cargas. Si esto ocurre, puede ponerse un puente en lugar del capacitor de acoplamiento C35. Otra solución es colocar una resistencia de descarga a través de Car+ y Car-. Finalmente, el uso de un transformador de acoplamiento de entrada en la carga previene un recorte no deseado en la salida del McVCO.

\section{$\mathrm{J} 1 \mathrm{y} \mathrm{J} 2$}

Las conexiones opcionales de entrada y salida están provistas a través de los conectores $\mathrm{J} 1$ y J2. Estos son receptáculos modulares de ocho pins. Ver Figura $2 B$ para los funciónes de los pins. J1 es una conexión de salida para la alimentación y la subportadora de audiofrecuencia. Cuando un transmisor es colocado en el mismo gabinete que el $\mathrm{McVCO}$, el cable de teléfono logra una conexión apropiada. J2 provee una conexión de entrada para las subportadoras de audiofrecuencia generadas por otro equipo. La etapa de salida del McVCO es un amplificador sumador y las señales que aparecen en J2 son sumadas con la señal generada por el McVCO.

Utilizando $\mathrm{J} 1$ con $\mathrm{J} 2$ puede instalarse fácilmente un sismómetro de tres componentes. Los tres McVCOs están encadenados de manera que la salida $\mathrm{J} 1$ de la primer tarjeta va conectada a la entrada J2 de la segunda. El J1 de la segunda tarjeta va conectado al J2 de la tercera. La salida de la tercer tarjeta tendrá entonces las subportadoras para los tres componentes. Para más detalles, ver Parte III, Conexión de tres componentes.

\section{Sockets $\mathrm{S}$ y $\mathrm{T}$}

Para calibrar la respuesta del sismómetro con un atenuador en la entrada del amplificador de la señal sísmica, se cuenta con un socket para las resistencias S y T, ver Figura $2 B$. Para preservar el rechazo a ruido en modo común, la resistencia $T$ es dividida en dos partes iguales, que se convierten en las resistencias T1 y T2. Las resistencias T están ubicados en serie con las conexiones de entrada I+ e I- y el amplificador de entrada. Si las resistencias T no son utilizados 
Parte I. Funcionamiento del McVCO

o se encuentran ubicadas fuera de la tarjeta, entonces cada posición de la resistencia $\mathrm{T}$ del socket debe ser puenteado con un alambre o la entrada se desconectará.

Debe tenerse cuidado al soldar y alambrar el socket. Una conexión abierta o intermitente arruinaría toda la instalación. 
Parte I. Funcionamiento del McVCO

\section{Funcionamiento en el Campo}

Todo lo que se necesita para poner en funcionamiento el McVCO es conectar la alimentación, el sismómetro y la salida y ajustar el interruptor DIP para la ganancia y la frecuencia deseada. El tema de las conexiones es tratado en la sección titulada Notas sobre la Conexión, y el ajuste del interruptor DIP en la sección Prueba en banco. Las Figuras $2 A$ y $2 B$ resumen esta información.

Esta sección cubre dos temas: cómo encontrar el ajuste correcto de ganancia, y qué hacer si el $\mathrm{McVCO}$ no funciona inmediatamente dentro de los dos o tres $\mathrm{Hz}$ de la frecuencia central nominal.

\section{Ganancia}

Si el McVCO está reemplazando a otro instrumento, entonces todo lo que se necesita es traducir el ajuste de la ganancia del instrumento anterior al del McVCO. Al realizar esto se presenta una complicación debido a que muchos instrumentos anteriores utilizan atenuadores cuyo ajuste debe ser restado de la ganancia no atenuada del instrumento para encontrar la ganancia de operación. Por ejemplo, el USGS J502 tiene una ganancia no atenuada de $90 \mathrm{~dB}$. Si el atenuador es ajustado a $18 \mathrm{~dB}$, entonces la ganancia es $72 \mathrm{~dB}(90 \mathrm{~dB}-18 \mathrm{~dB})$. El ajuste de la ganancia del McVCO para la misma respuesta es de $72 \mathrm{~dB}$.

Si no puede encontrar el ajuste de ganancia anterior o si el McVCO está siendo instalado en un nuevo lugar, entonces el nivel y ajuste de ganancia debe ser obtenido a partir de la observación del ruido sísmico de fondo. TP2 está provisto para ver la señal sísmica con un osciloscopio. La señal en TP2 es similar a la señal a la salida del discriminador. Tiene la fase opuesta pero aproximadamente la misma amplitud. La amplitud en el discriminador dependerá de la sensibilidad de modulación y demodulación.

Conecte un osciloscopio a TP2 y halle el ajuste de ganancia que dé un nivel de señal de ruido de fondo aceptable. Una buena ganancia para comenzar es $66 \mathrm{~dB}$, y muchos sitios son ajustados para una señal de fondo de pico a pico de $50 \mathrm{a} 100 \mathrm{mV}$. Recuerde de dar un reset luego de cada cambio de los interruptores DIP y espere un minuto para que finalicen el ajuste automático a cero y las rutinas de prueba.

\section{Frecuencia}

Muy rara vez el ajuste automático a cero, después de un reset, fallará para nivelar f1 dentro de 2 $\mathrm{Hz}$ de la frecuencia central nominal. Esto generalmente ocurre porque ocurrió una señal grande al final del período de la puesta automática a cero, o la señal sísmica de fondo tiene componentes grandes de largo período. La solución es simple. De un reset a la unidad y evite moverla durante el período siguiente de 10 segundos de ajuste automático a cero. Un par de intentos deberían lograr una buena frecuencia central, incluso con la presencia de fondo de señales de largo período.

Si el McVCO está instalado dentro de un gabinete con un transmisor y funciona con mucha ganancia, la frecuencia central puede cambiar cuando se cierre la tapa del gabinete. La dispersión de la onda portadora de radio en los circuitos del McVCO generalmente aparece como un componente de CD en el amplificador de señal sísmica. Si sospecha de este problema, trate de 
Parte I. Funcionamiento del McVCO

cerrar la tapa del gabinete y de interrumpir la alimentación para provocar un ajuste automático a cero y un reset con el radio y el McVCO en condiciones operativas.

Si el transmisor es encendido a través de $\mathrm{SW}+$, la unidad se ajustará automáticamente a cero antes de que el transmisor se encienda. Esto puede causar un offset temporal pero el sistema de corrección de offset de largo plazo colocará la frecuencia promedio f1 en la frecuencia del canal central nominal. Por lo tanto no se preocupe si no tiene tiempo, el McVCO se autoajusta.

Existen dos situaciones por las cuáles un McVCO funcional podría fallar en el ajuste automático a cero. En algunos sitios en donde la antena en el mástil no está conectada a tierra, el cable de entrada del sismómetro puede captar y rectificar suficiente energía de RF como para saturar al amplificador de señal sísmica. Si el McVCO se pone en cero cuando el transmisor está apagado, el problema se deberá a una mala conexión a tierra en la antena. Arréglelo.

La otra causa de los problemas con el ajuste automático a cero es la corrosión del sismómetro. En particular, la corrosión en donde los conductores de la bobina que pasan por la cubierta del sismómetro L4 puede crear una celda electroquímica lo suficientemente potente como para desequilibrar al amplificador de la señal sísmica. Si el McVCO se pone automáticamente en cero con el sismómetro desconectado, piense que podría ser una celda corroida. La presencia de la misma es detectada por un voltaje de CD entre cualquiera de los lados del sismómetro o la bobina. Las salidas de las celdas de corrosión son intermitentes y sensibles a la temperatura.

E1 McVCO posee un amplificador de señal sísmica acoplado directamente. El voltaje de CD aplicado a las entradas del amplificador aparece en la salida del amplificador como un offset. Los offsets son corregidos por el lazo de realimentación de corrección de offset, pero el voltaje de entrada de CD máximo que el circuito puede anular es de $\pm 0.5 \mathrm{mV}$.

Desafortunadamente, las celdas de corrosión pueden producir decenas de milivoltios a la entrada del amplificador de señal sísmica. Esto es suficiente para saturar la etapa de entrada en la mayoría de los amplificadores de señal sísmica, incluso cuando las etapas posteriores sean acopladas en forma capacitiva.

Si la celda de corrosión está inactiva, la corrosión puede ser detectada con un ohmmetro. El fabricante del sismómetro L4, Mark Products, específica una fuga entre la bobina y el cuerpo del L4 como mayor a $100 \mathrm{M} \Omega$ en 500 volts. Las celdas de corrosión producen lecturas de resistencia menores a los $10^{6} \Omega$ con un ohmmetro común. La desconexión del blindaje del cable del sismómetro o de la conexión a tierra del McVCO podría desconectar la celda si la corrosión se encuentra solamente en un lado de la bobina. Sin embargo, una vez que la corrosión comienza en un lado de la bobina, generalmente se desarrolla también en el otro lado y termina en el circuito de entrada del amplificador. Los problemas en las celdas de corrosión pueden ser intermitentes, pero no se arreglan solos. Por lo general la corrosión crece y el problema empeora. Los sismómetros con signos de corrosión deben cambiarse.

En una situación difícil, el margen de ajuste de offset del instrumento puede incrementarse en un factor de 4. Para incrementar este margen, corte el conductor para R20 en el borde superior de la tarjeta. Esto elevará el voltaje de referencia para el circuito de anulación del desbalance de 50 a 
Parte I. Funcionamiento del McVCO

200 milivolts. El ajuste automático a cero tenderá a excederse cuando el instrumento sea reiniciado y el ruido de la anulación del offset podría incrementarse. Sin embargo, el gran margen de anulación de offset podría funcionar bien como una medida temporal para la reparación de un sitio que necesita una mejor conexión a tierra de la antena, o un sismómetro sin corrosión interna.

La subportadora del segundo canal, f2, no forma parte de los sistemas de ajuste automático a cero o de corrección de offset. Cuando se la utiliza, es un canal de poca ganancia y por lo tanto es estable. Si fuera necesario, el potenciómetro de ajuste Shift 2, P4, puede utilizarse para ajustar f2 a la frecuencia central del canal. 
Parte I. Funcionamiento del McVCO

\section{Procedimiento de Setup}

El funcionamiento correcto del McVCO depende del ajuste de los circuitos para el cambio de nivel y el punto cero del amplificador de señal sísmica. Estos ajustes establecen el punto de operación alrededor del cual el ajuste automático a cero y los circuitos de corrección del offset funcionan. El ajuste incorrecto disminuye la capacidad de estas rutinas para mantener el funcionamiento en el centro del canal.

El ajuste debe realizarse alrededor de la temperatura en la que se espera que la unidad funcione. La temperatura ambiente es generalmente la más adecuada. Para revisar el ajuste en la mesa de pruebas antes de llevar la unidad al campo sólo se necesitan unos minutos y es conveniente hacerlo. Se necesita un voltímeto digital DVM de 3 y medio dígitos.

Ver Figura $2 A$ para la ubicación de los ajustes.

1) Coloque la unidad en el modo de apagado o suspención ajustando los interruptores DIP de modo que $\mathrm{fl}=\mathrm{f} 2$ y ajuste la ganancia para $66 \mathrm{~dB}$.

2) Suministre energía o de un reset a la unidad

3) Conecte un DVM entre GND y TP1 y ajuste el potenciómetro Null, P1, (punto de cero o cancelación), hasta leer $0 \mathrm{VCD} \pm 10 \mathrm{mV}$.

4) Conecte un DVM entre GND y TP3 y ajuste Shift 1, P3, hasta leer $2.50 \mathrm{VDC} \pm 10 \mathrm{mV}$.

5) Conecte un DVM entre GND y TP4 y ajuste Shift 2, P4, hasta leer $2.50 \mathrm{VDC} \pm 10 \mathrm{mV}$.

Esto completa el ajuste de setup. 
Parte I. Funcionamiento del McVCO

\section{Prueba de Calibración}

La calibración puede revisarse y ajustarse con un equipo simple. Se requieren un multímetro digital DVM de tres dígitos y medio; un contador de frecuencia de rango audible y una fuente de alimentación de 1.5 VCD como la utilizada en la sección de la Prueba de Banco de este manual. No ajuste la calibración sin completar la prueba de calibración.

1) Para realizar la calibración es necesario conocer la sensibilidad de desviación del modulador para su red sísmica dada en $\mathrm{Hz} / \mathrm{V}$. Averigüe esto antes de continuar. Por ejemplo, el J502 y J512 producidos por el USGS utilizan $115 \mathrm{~Hz} / 4.05 \mathrm{~V}$ y los McVCO utilizan esta sensibilidad de desviación para las instalaciones del USGS. Otra sensibilidad frecuente de desviación es de 125 $\mathrm{Hz} / 3.00 \mathrm{~V}$.

2) Prepare una fuente de $1.5 \mathrm{mVCD}$ conectando resistencias de $100 \Omega \pm 1 \%$ y $100 \mathrm{~K} \Omega \pm 1 \%$ a través de una batería de $1.5 \mathrm{VCD}$. Conecte un extremo de la resistencia de $100 \Omega$ al lado negativo de la batería. Conecte un extremo de la resistencia de $100 \mathrm{~K} \Omega$ al lado positivo de la batería. Junte los extremos sueltos de las resistencias. La fuente de $1.5 \mathrm{mVCD}$ está a través de la resistencia de 100 $\Omega$.

3) Mida el voltaje de la batería de la fuente de $1.5 \mathrm{mV}$. Las resistencias dividen el voltaje de la batería por 1000. Cuando la ganancia de la unidad es regulada para $60 \mathrm{~dB}$ (1000), el cambio en la frecuencia $(\Delta \mathrm{f})$ es (voltaje de la batería) x (sensibilidad de desviación). Haga este cálculo.

4) Si la unidad utiliza un socket con resistencias $T$, reemplace este con uno que tenga puentes de unión en las posiciones T1 y T2 del socket. La calibración de la ganancia está referenciada a la entrada del amplificador de la señal sísmica y no incluye la atenuación del socket $\mathrm{S}$ y $\mathrm{T}$. Ver la Parte III, "Respuesta en Frecuencia y Ganancia" para mayor información.

5) Ajuste la unidad para una ganancia $=60 \mathrm{~dB}$. Puede ajustarse cualquier valor de la frecuencia central fl puede regularse ya que la calibración es válida para cualquier frecuencia seleccionada. Si se utiliza un multímetro DVM con contador de frecuencias, seleccione un valor bajo de f1 para incrementar al máximo la resolución de su medida de f1. No intente regular la calibración si no puede hacer una medida de frecuencia con una resolución de $1 \mathrm{~Hz}$.

6) Conecte los puntos de prueba a las entradas del sismómetro I+ e I- y una los extremos sueltos. Suministre energía a la unidad o de un reset. Espere un minuto para que el ciclo de prueba y el ajuste automático a cero terminen y registre la frecuencia central de funcionamiento (fc) medida en TP5. Esta debe estar $2 \mathrm{~Hz}$ alrededor de la frecuencia f1 seleccionada.

7) Calcule la desviación negativa y positiva de f1 del valor de $\Delta \mathrm{f}$ en el paso 3 y fc en el paso 6.

$$
\begin{aligned}
& +D e v=f c+\Delta f \\
& -D e v=f c-\Delta f
\end{aligned}
$$


Parte I. Funcionamiento del McVCO

8) Con los puntos de prueba suministre la fuente de $1.5 \mathrm{mVCD}$ a la entrada del sismómetro. Conectando el lado negativo de la batería a I- con I+ alambrado a la conexión entre las dos resistencias, da una desviación positiva. Revertiendo la conexión da como resultado una desviación negativa. Compare las desviaciones medidas con las calculadas.

9) Las desviaciones calculadas y medidas deben estar dentro de $1 \mathrm{~Hz}$. Si fuera necesario ajuste el potenciómetro de calibración, Cal, P2 y repita la prueba para la otra polaridad de entrada. Ajuste hasta que ambas desviaciones estén dentro de $1 \mathrm{~Hz}$ de sus valores calculados. Comience nuevamente el paso 6 si el ajuste no da resultado. Cada vez que repita este proceso, no es necesario el reset en el paso 6, pero es importante que revise la frecuencia central con la entrada en corto circuito.

Este método da una exactitud de calibración de hasta un 3\% en todos los márgenes de ganancia y es independiente de la frecuencia $\mathrm{fl}$. Se puede obtener un ajuste mejor con una referencia de 2.5 $\mathrm{mV}$ atenuada a $60 \mathrm{~dB}$ en lugar de la batería y las resistencias. No se provee ningún ajuste para la calibración del segundo canal definido por el usuario. 


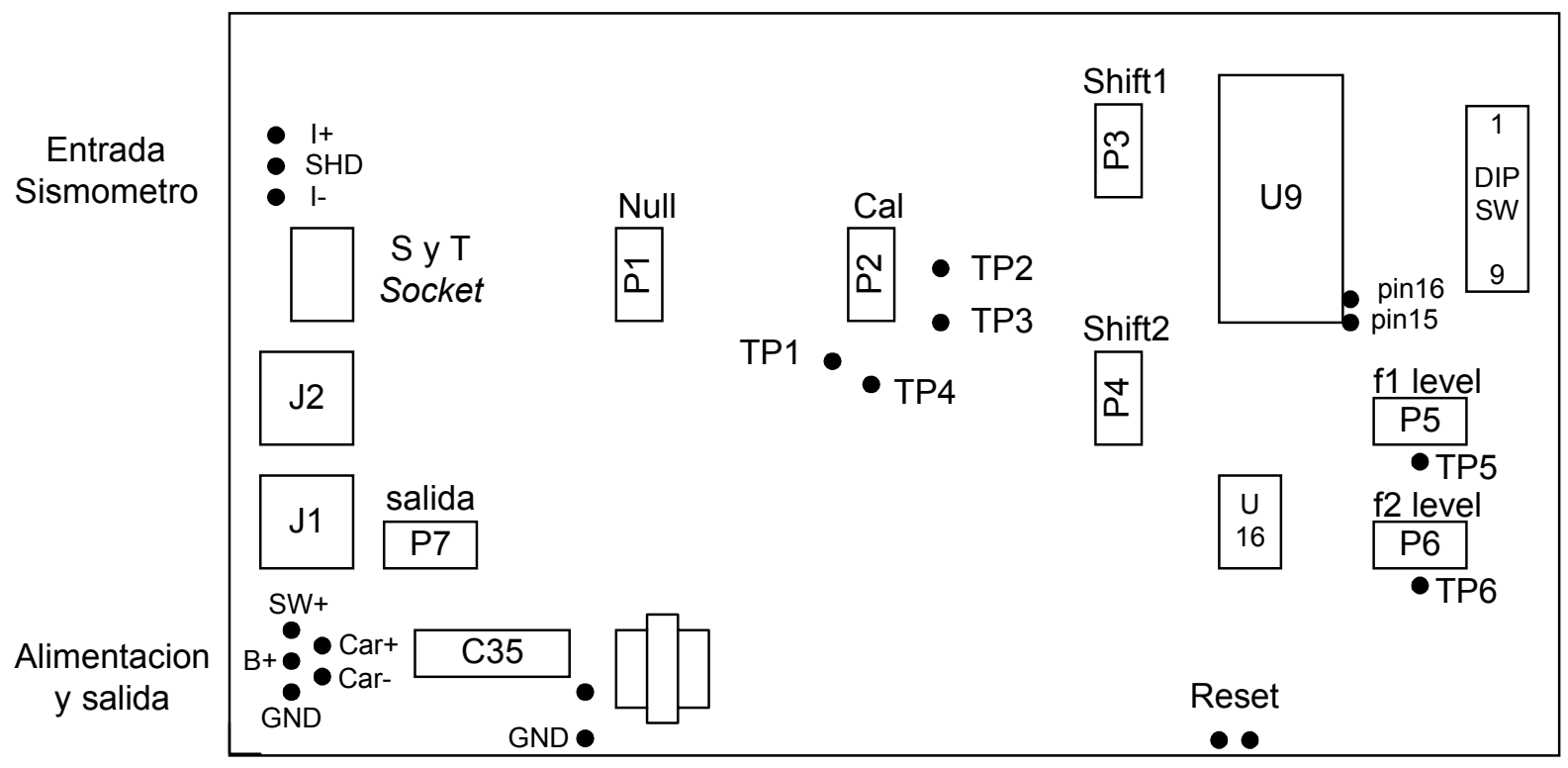

E/S Rotulos

$\mathrm{B}+$ : conexion al alimentacion de $12 \mathrm{v}$

GND :conexion al tierra del fuente, $0 \mathrm{v}$

SW+ : salida de potencia conmutada de 12v

Car+ and Car- : salidas de la subportador

I+ : conexion al lado positivo del sismometro

I- : conexion al lado negativo del sismometro

SHD : conexion al blindaje del cable sismometro.

\section{Posiciones del interruptor DIP para seleccion de frecuencias y ganancias}

OJO:

\begin{tabular}{|c|c|cc|}
\hline $\begin{array}{c}\text { GANA } \\
(\mathrm{dB})\end{array}$ & \multicolumn{2}{|c|}{ SW } \\
\hline 1 & 2 & 3 \\
\hline 42 & 0 & 0 & 0 \\
48 & 0 & 0 & 1 \\
54 & 0 & 1 & 0 \\
60 & 0 & 1 & 1 \\
66 & 1 & 0 & 0 \\
72 & 1 & 0 & 1 \\
78 & 1 & 1 & 0 \\
84 & 1 & 1 & 1 \\
\hline
\end{tabular}

\begin{tabular}{|c|c|ccc|}
\hline $\mathrm{f} 2$ & \multicolumn{2}{|c|}{ SW } \\
$(\mathrm{Hz})$ & 7 & 8 & 9 \\
\hline 680 & 0 & 0 & 0 \\
1020 & 0 & 0 & 1 \\
1360 & 0 & 1 & 0 \\
1700 & 0 & 1 & 1 \\
2040 & 1 & 0 & 0 \\
2380 & 1 & 0 & 1 \\
2720 & 1 & 1 & 0 \\
3060 & 1 & 1 & 1 \\
\hline
\end{tabular}

Cambios al interruptores DIP no tomen efecto antes que de un reset al unidad.

Modo de suspencion sucede cuando interruptores 4,5,6 egual $7,8,9$

Figura 2A. Ubicación de las partes y del Interruptor DIP. 


\section{J1 y J2 conectores modulares}

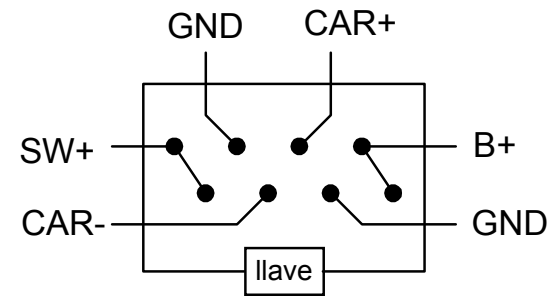

(visto de abajo)

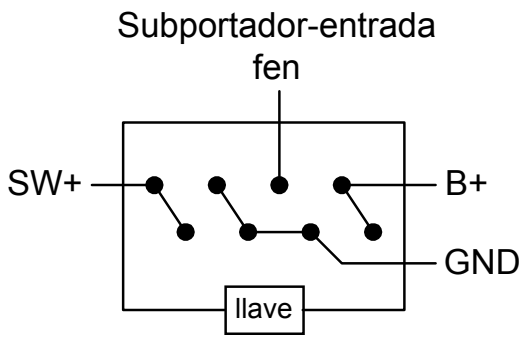

(visto de abajo)

J1: salidas opcionales

J2: entrada subportadores

Se puede inteconectar las tarjetas McVCO por medio de J1 y J2 para construir instrumentos de varios componentes

Ver Figura $2 \mathrm{~A}$ para E/S rotulos

\section{Socket Resistencias S y T}
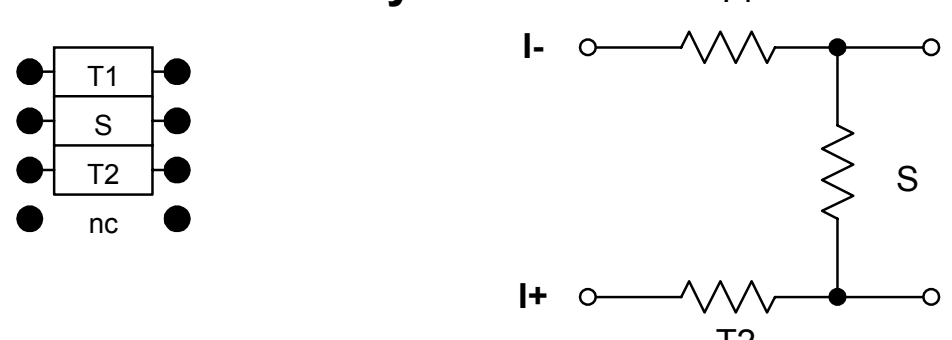

$\mathrm{T} 2$

Si las resistencias $\mathrm{T}$ no son utilizados o se enquentran ubicadas fuera de la tarjta, entonces ambos posiciones de las resistencias T1 y T2 deben ser puenteado con un alambre o la entrada se desconectara.

\section{Puntos de prueba y ajustes}

Ver Figura 2A para lugares.

TP1 : punto de cero del preamplificador en modo de suspencion

TP2 : senal sismica con respeto al nivel de $0 \mathrm{v}$

TP3 :entrada al canal 1 del CAD, con respeto al nivel de $2.5 \mathrm{v}$

TP4 :entrada al canal 2 del CAD, con respeto al nivel de $2.5 \mathrm{v}$

TP5 :subportador $\mathrm{f1}$. Se puede ajustar este nivel con P5

TP6 :subportador f2. Se puede ajustar este nivel con P6

P7 :ajuste salida de todos subportadores

No tentar de ajustar P1, P2, P3, P4 sin seguir el Procedimiento de Setup.

NO SEA NECESARIO AJUSTAR P1, P2, P3, P4 EN EL CAMPO

Figura 2B. Pins de salida y entrada y puntos de prueba. 


\section{Parte II: Referencia Técnica}




\section{Descripción del Circuito}

Esta descripción es para utilizarse con los diagramas esquemáticos del McVCO incluidos al final de esta sección como Figura $3 A, 3 B, 3 C$. La descripción aplica a las tarjetas del circuito impreso nominados "MCFM05" y "McVCO01". El orden de los pins de los circuitos integrados está dado con el pin 1 en la esquina superior izquierda y el último pin en la esquina superior derecha.

\section{Entrada/Salida y Suministro de CD (Figura $3 A$ )}

En la parte inferior izquierda del diagrama esquemático se encuentran dibujados los pins de conexión para soldar la alimentación y la frecuencia portadora de salida. Los mismos están nominados "SW+", "CAR+", "B+", "CAR-“, y "GND”.

B+ es la conexión positiva de una fuente de alimentación o batería de 12 VCD.

GND es la conexión negativa para la fuente de alimentación y es tierra, el punto de referencia de 0 volts. Este esquema a veces muestra esta conexión con el símbolo de tierra o lo indica como "OV".

El lado positivo de la fuente de alimentación pasa por el diodo de protección contra la inversión de la polaridad, D1, hasta los circuitos de fuente conmutada formados con U14, Q1, Q2 y Q3. La razón principal para la fuente conmutada es lograr un reset limpio y sin fallas para el microcontrolador. Por ejemplo, en determinadas circunstancias, los sistemas de energía solar pueden acercarse gradualmente al voltaje de funcionamiento y fallar el reset del

microcontrolador. Esto provoca un comportamiento impredecible, incluyendo la producción de tonos de salida con interferencia. La fuente conmutada elimina el problema. Otro uso secundario de la fuente conmutada es alimentar una carga externa.

El interruptor primario está formado por U14, un amplificador operacional conectado como un comparador. El voltaje interno de referencia en el pin 2 es comparado con una fracción de la fuente de alimentación positiva en el pin $3 \mathrm{y}$ la salida en el pin 6 cambia al voltaje positivo de entrada $(\mathrm{V}+)$ o a GND. R35 provee retroalimentación positiva para una histéresis de un volt para que el circuito se encienda $(\mathrm{V}+)$ con " $\mathrm{B}+$ " a 11.5 volts y se apague $(0 \mathrm{~V})$ cuando "B+" sea alrededor de 10.5 volts.

Para la versión MCFM05, la salida de U14 se divide entre el interruptor de carga externa formado por Q2 y Q3, y hacia el circuito seguidor de voltaje, Q1. E1 interruptor de carga externa sale en "SW+". Se deben conectar con cuidado las cargas externas para reducir la resistencia hacia la batería. Si el voltaje de la batería es baja, las caídas de voltaje provocadas por las pérdidas IR pueden superar la histéresis de U14 y el interruptor oscilará. El circuito RC en la compuerta de Q2 provee un retardo en la acción del interruptor mientras se estabilizan las cargas externas. 
En la versión McVCO01, la salida "SW+" es conmutada por el microcontrolador a través de Q2 y Q3. E1 interruptor se enciende $10 \mathrm{seg}$ luego de ser alimentada la unidad o de darse un reset. Cuando se enciende "SW+", si el voltaje de la batería es baja y las pérdidas IR en la conexión desde la batería son mayores que la histéresis del comparador, éste desconectará la alimentación. Con la alimentación desconectada, el voltaje de la batería se recupera y el McVCO se enciende otra vez. Diez segundos después, el microcontrolador encienda la carga. Nuevamente, el voltaje de la batería cae hasta alcanzar el nivel de limitación inferior del comparador y la unidad se apaga. De este modo, la salida de la subportadora parecerá estar "congelada" en la frecuencia central del canal y un pulso muy leve aparecerá en "SW+". Para más información, ver Parte III, “Utilización de la Potencia de Salida Conmutada".

La fuente de alimentación regulada recibe su voltaje de entrada desde el emisor de Q1. Esta es aproximadamente el voltaje de salida del comparador U14 hasta que excede el voltaje del diodo zener de 15 VCD, D2. Luego el emisor de Q1 es fijado en 14.4 VCD. El zener y el supresor de voltajes transitorios VS4, proveen protección contra las sobrecargas. Un voltaje mayor de 20 VCD a una corriente reducida es posible cuando los paneles solares de 12 VCD son descargados por las baterías. VS4 limita los paneles solares sin carga hasta más o menos $20 \mathrm{VCD}$ y puede derivar 100 mA indefinidamente. VS4 con VS1, VS2, VS3, VS5, VS6 y VS7 proveen protección al transitorio de voltaje en las entradas y salidas del instrumento.

U12 es un regulador de $+5 \mathrm{VCD}$ con un comparador interconstruido. El comparador se utiliza para proveer un pulso de reset para el microcontrolador. Este reset es iniciado por un cortocircuito a través de R40 en los dos pins de reset provistos. U13 es un inversor de conmutación que convierte los +5 VCD desde U12 a -5 VCD. Estos dos suministros abastecen de alimentación al resto de los circuitos del instrumento.

La portadora modulada de audio sale de los pins rotulados "CAR+" y "CAR-" hasta 4V p-p hacia $600 \Omega$ a través del transformador T1. C35 bloquea las fuentes externas de CD. R32 eleva la impedancia primaria para lograr un acoplamiento de impedancias de $600 \Omega$ y con C21 se aisla la sección del amplificador de salida del amplificador operacional cuádruple U3. El amplificador de la portadora de salida es un inversor sumador cuya ganancia varía desde 2 a 1 con el ajuste del potenciómetro de nivel de salida Output, P7. Las entradas para el amplificador sumador provienen de las dos salidas del generador de ondas f1 y f2 (ver Figura $3 \boldsymbol{C}$ ) y de una portadora de audio externa, fen, que puede ser conectada a través de $\mathrm{J} 2$.

$\mathrm{J} 1$ y $\mathrm{J} 2$ son jacks modulares mostrados en el borde izquierdo de la Figura $3 \boldsymbol{A}$. Las conexiones de $\mathrm{J} 1$ son las mismas que las de los pins de conexión para soldar excepto para la versión MCFM05. Para MCFM05, "B+" en los jacks modulares es tomado desde el cátodo de D1, el diodo de protección contra inversión de polaridad de entrada. J2 provee energía no regulada, conexión a tierra, y una conexión de entrada portadora fen. J1 puede utilizarse para conectar al instrumento a un radiotransmisor. $\mathrm{J} 2$ conecta y alimenta a otros instrumentos telemétricos. Utilizando juntos $\mathrm{J} 1$ y $\mathrm{J} 2$ se puede conectar una estación sísmica de tres componentes encadenando tres McVCO. Para más detalles ver Parte III, “Conexión de Tres Componentes”. 
Por encima de $\mathrm{J} 2$ el diagrama muestra la conexión de la entrada del sismómetro al socket de $\mathrm{S}$ y T. Los conductores de la bobina del sismómetro están conectados a "I+" y "I-“" con la polaridad indicada. El cable blindado del sismómetro puede conectarse a tierra a través de la conexión provista. Desde estos bornes para soldar, la entrada va un socket hembra de 8 pins en donde se pueden insertar las resistencias S y T. La resistencia T está dividida en dos componentes iguales: T1 y T2 para preservar el rechazo a ruido de modo común a la entrada. Si las resistencias T no se utilizan o no están ubicados en el socket, entonces T1 y T2 deben ser reemplazados por puentes para completar la conexión de entrada. Para realizar pruebas de calibración por impulsos, R7 conecta el sismómetro al pin de entrada/salida del microcontrolador. Desde el socket, las conexiones se realizan al amplificador de la señal sísmica mostrado en la Figura $\mathbf{3 B}$.

La parte superior de la Figura $\mathbf{3 A}$ muestra los componentes que forman el segundo canal opcional. Estos componentes y una pequeña área de prototipo junto a U3 permiten a los usuarios construir sus propios circuitos, ver Parte III, Utilización del Segundo Canal de Poca Ganancia.

La versión del McVCO de ganancia dual del USGS utiliza los componentes opcionales como se muestra en el centro de la Figura $\mathbf{3 A}$. Forman una interfaz fija de baja ganancia desde el filtro (Figura 3B) hacia el segundo canal del conversor analógico-digital (CAD) (Figura 3C). Esto permite realizar y enviar por telemetría mediciones de alta y baja ganancia de la misma señal sísmica. La señal sísmica es invertida por U3 en el pin 1 y luego cambiada por U15 a un nivel de polarización de $2.5 \mathrm{VCD}$, apropiada para la entrada del conversor analógico-digital de 0 a 5 volts. R48 es seleccionada para atenuar el rango máximo de $10 \mathrm{~V}$ pico-pico en el pin 1 de U3 y para calibrar la ganancia total del canal. El valor mostrado de $1.8 \mathrm{M} \Omega$ da la misma desviación de frecuencia que un USGS J502 con una sensibilidad de desviación de $115 \mathrm{~Hz} / 4.05 \mathrm{~V}$ operada a una ganancia de $40 \mathrm{~dB}$ (atenuación $=50 \mathrm{~dB}$ ).

\section{Amplificador de Señal Sísmica (Figura 3B)}

La señal del socket con S y T (Figura 3A) se aplica al amplificador de instrumentación U1 a través del circuito de entrada en In- e In+ (Figura $3 B$ ). Dado que la bobina del sismómetro no está referida a tierra, R1 aparece en serie con R2 a través de la bobina para lograr la impedancia de entrada estándar de $10 \mathrm{~K} \Omega$. R3 y R4 son resistencias limitadoras de corriente que protegen a U1. Algunas conexiones mostradas son para un relevador FL1, que no se utiliza en esta aplicación. R5 y R6 reducen la ganancia de U1 y no se utilizan aquí. La ganancia de U1 es 100 $(40 \mathrm{~dB})$.

Un filtro de 2 etapas sigue al amplificador de instrumentación de entrada. Está formado por dos amplificadores operacionales del amplificador cuádruple U2. El filtro de 4 polos tiene una respuesta Butterworth de pasa bajas de $30 \mathrm{~Hz}$ y es de ganancia unitaria. La salida del filtro en el pin 8 de U2 se puede utilizar para el segundo canal de baja ganancia y es la entrada del amplificador de ganancia variable. 
El circuito amplificador de ganancia variable mostrado en la parte inferior de la Figura 3B, está formado por el conversor digital-analógico (CDA) U4, y una sección de U3, un amplificador operacional cuádruple. U4 es un CDA multiplicador de 12 bits y es utilizado como el elemento de retroalimentación en el amplificador inversor implementado con U3. A través de las líneas de interfaz serie "Data", "Clock" y "Gain" el microcontrolador (Figura 3C) fija la amplificación de esta etapa para proveer la ganancia seleccionada por los interruptores DIP. El control de ajuste a cero o cancelación (Null) P1 se utiliza para poner a cero al amplificador de ganancia variable en TP1 cuando el instrumento está en el modo de apagado (ver Ajuste Inicial en la Parte I). La salida referenciada a cero tiene una rango pico-pico de casi $10 \mathrm{~V}$ que debe reducirse para la conversión analógica a digital de 0 a 5 volts. El potenciómetro Cal, P2, provee la atenuación necesaria y ajusta la calibración del instrumento (ver Prueba de Calibración en la Parte I). TP2 es el punto de prueba donde la señal sísmica amplificada puede verse.

La salida bipolar en TP2 debe nivelarse a 2.5 VCD para el CAD. Esto se produce en la sección del amplificador operacional de U5, en donde la señal es sumada con un nivel de CD desde el potenciómetro Shift 1, P3. En TP3, la señal sísmica abarca un rango de 0 a 5 volts referenciada a $2.5 \mathrm{VCD}$ según lo requiere la conversión analógica a digital. La salida de U5 en el pin 6 se conecta al primer canal de CAD en el pin 2 de U6 (CH0). Ver Figura $3 \boldsymbol{C}$.

El microcontrolador ajusta el offset y la ganancia del amplificador de señal sísmica. Otro CDA de 12 bits, U7, se encuentra conectado al controlador a través de las líneas de interfaz serie "Data", "Clock" y "Offset" (ver el centro de la Figura 3B). La sección de referencia de U5 produce 200 $\mathrm{mVCD}$ que se dividen $50 \mathrm{mVCD}$ por R19 y R20 para la entrada de referencia de CDA, U7 pin 1. El CDA proporciona una corriente en el pin 3 que es producto del conteo del CDA por el voltaje en el pin de referencia. En el pin 14 de U2 la corriente del CDA se cambia a una voltaje que se suma con el voltaje de referencia de $50 \mathrm{mV}$. Consecuentemente la salida de U2 en el pin 1 es una voltaje entre $\pm 50 \mathrm{mV}$ que es proporcional al conteo del CDA. Este voltaje se aplica a la terminal de tierra del amplificador de instrumentación, pin 1, U1 y es utilizada por el microcontrolador para ajustar el nivel de CD en la salida del amplificador de señal sísmica, U5 pin 5.

\section{CAD, Microcontrolador y Generador de Ondas Senoidales (Figura 3C)}

Las frecuencias del reloj requeridas por el microcontrolador U9, derivan del oscilador de cristal de 4.096 MHZ mostrado en la parte inferior de la Figura 3C. La salida aislada del oscilador U10 pin 10, acciona una cadena divisora U11 que produce $1.024 \mathrm{MHZ}$ en el pin 7 para el reloj del microcontrolador U9 pin 27 y $1.0 \mathrm{KHz}$ en el pin 1 para la entrada del reloj de tiempo real U9 pin 1. Un reloj de $512 \mathrm{KHz}$ es producido por el divisor en el pin 6 para las entradas del reloj del generador de ondas senoidales, pin 8 en U8 y U16.

La mayor parte de la actividad del microcontrolador es transmitida a través de la interfaz serie formada por sus pins de entrada y salida. Las líneas comunes para el reloj serial, U9 pin 13, y los datos serie, U9 pin 14, conectan los CDAs de ganancia del amplificador de señal sísmica y offset 
y los generadores de forma de onda $\mathrm{fl}$ y f2. Como se muestra, las líneas individuales para la selección de dispositivos direccionan a cada circuito integrado.

El interruptor DIP, DS1 es leído en los pins 17 al 25 de las entradas y salidas del microcontrolador. El interruptor 1 está conectado al pin 25 con el interruptor 9 conectado al pin 17. La tabla del lado derecho de la Figura $3 C$ muestra cómo la ganancia y la frecuencia son seleccionadas por los interruptores. Los interruptores son "activos bajo"; es decir, un interruptor ON baja la línea que el microcontrolador interpreta como un 0 lógico. La condición no válida de f1 y f2 seleccionada para la misma frecuencia resultará en el apagado del instrumento. Este estado es utilizado para ajustar el amplificador de señal sísmica (ver Parte I, Ajuste Inicial) pero debe evitarse cuando el instrumento está en uso, incluso si la frecuencia del segundo canal f2, no es utilizada. DS1 es leído por el microcontrolador solamente después de un ciclo de encendido o reset.

Las cuatro líneas de entrada/salida restantes son utilizadas para diversos propósitos. La línea en el pin 6 está conectada a la bobina al relevador de entrada del amplificador de la señal sísmica RL1, que no se usa en esta aplicación. El pin 7 permite que se aplique un pulso al sismómetro a través de R7 (Figura 3A) como parte de la prueba periódica programada. Las líneas de entrada/salida en los pins 15 y 16 se utilizan como banderas para saltos del programa en algunas versiones del programa del microcontrolador, en donde 5 VCD en el pin 15 habilita la rutina de prueba periódica, y $5 \mathrm{VCD}$ en el pin 16 activa la operación con dos canales.

El conversor analógico a digital CAD, U6, responde a las demandas del microcontrolador para convertir el voltaje de entrada en cualquiera de los dos canales de entrada en los pins 2 ó 3 . Luego de que la conversión es transferida al microcontrolador, el CAD espera mientras el controlador transforma la medición en un código y lo envía a los generadores de forma de onda f1 o f2, U8 o U16. El generador utiliza el código para sintetizar la frecuencia de salida instantánea correspondiente a la medición del CAD y a la frecuencia de la onda portadora seleccionada. El ciclo comienza otra vez con una nueva medición del CAD y se repite aproximadamente 660 veces por segundo para un solo canal y más o menos la mitad de esta tasa para la operación de dos canales.

El potenciómetro f1 Level, P5, y el potenciómetro f2 Level, P6, ajustan las amplitudes de salida de los generadores de formas de onda, U8 y U6 en TP5 y TP6. Las portadoras moduladas en estos puntos de prueba están conectadas con el amplificador sumador de salida, ver Figura $\mathbf{3 A}$.

Durante el ciclo de medición, el microcontrolador lea el reloj de tiempo real. En el intervalo correcto se realiza un ajuste al CDA de offset (Figura 3B) y los registros del temporizador de 24 o 18 horas son incrementados y verificados para un fin del ciclo de tiempo. Si ha sido seleccionado, al ocurrir el "timeout"la rutina programada de prueba es habilitada.

Al final de la misma, el ciclo de medición se reanudará.

En el encendido, o luego de un reset, el instrumento entrará en un modo de ajuste automático a cero en que $\mathrm{f} 1 \mathrm{y} f 2$ son ajustados a sus frecuencias portadoras centrales, mientras que el offset es 
eliminado de la salida del amplificador de la señal sísmica ajustando el conteo del CDA de offset. El ajuste automático a cero dura 10 segundos.

En la versión del McVCO01 del instrumento, la rutina programada de prueba es iniciada inmediatamente luego del ajuste automático a cero. Esta rutina dura alrededor de 42 segundos y consiste de $10.25 \mathrm{seg}$ de modulación de $21.25 \mathrm{~Hz}$ de la subportadora, seguidos por 18 seg para la prueba de pulsos del sismómetro, y luego por 13 seg de pulsos que proveen el ajuste de la ganancia y el ID (número de identificación) del instrumento. Un impulso positivo es "1" y uno negativo es "0". Los primeros tres pulsos dan la puesta del interruptor DIP de ganancia en el último reset cuando la ganancia fue puesta. Los últimos 10 impulsos son la representación binaria del número ID programado en el microcontrolador. Ver Parte III, "Ciclo de Prueba del McVCO" para más detalles.

\section{Diagramas Esquemáticos: Figura 3}

Las siguientes tres páginas contienen diagramas esquemáticos del McVCO.

\section{Notas para Figura 3a, marcado "Sheet 1 of 3":}

* indica componente del segundo canal opcional.

** El valor de R48 se selecta como lo mejor compromiso entre ganancia y rango dinamico. El valor mostrado de $1.8 \mathrm{M} \Omega$ da una ganancia total de $40 \mathrm{~dB}$ desde la entrada del preamplificador al salida del generador de ondas senoidales para una sensibilidad de desviacion de $115 \mathrm{~Hz} / 4.05 \mathrm{~V}$, cual es el patrone de sensibilidad de modulación del USGS para el J512.

1) El orden de los pins de los circuitos integrados está dado con el pin 1 en la esquina superior izquierda y el último pin en la esquina superior derecha.

Notas para Figura 3b, marcado "Sheet 2 of 3":

* RL1 mostrado pero no installado

** R5 y R6 no installado en ésta applicación

1) El orden de los pins de los circuitos integrados está dado con el pin 1 en la esquina superior izquierda y el último pin en la esquina superior derecha.

Notas para Figura 3c, marcado "Sheet 3 of 3":

* indica componente del segundo canal opcional.

1) Ganancias mostrados incluyen la sensibilidad de desviación y pueden estar probado por medir f1 en TP5 si la sensibilidad calibrado sea conocido.

2) El orden de los pins de los circuitos integrados está dado con el pin 1 en la esquina superior izquierda y el último pin en la esquina superior derecha. 
Parte II: Referencia Técnica

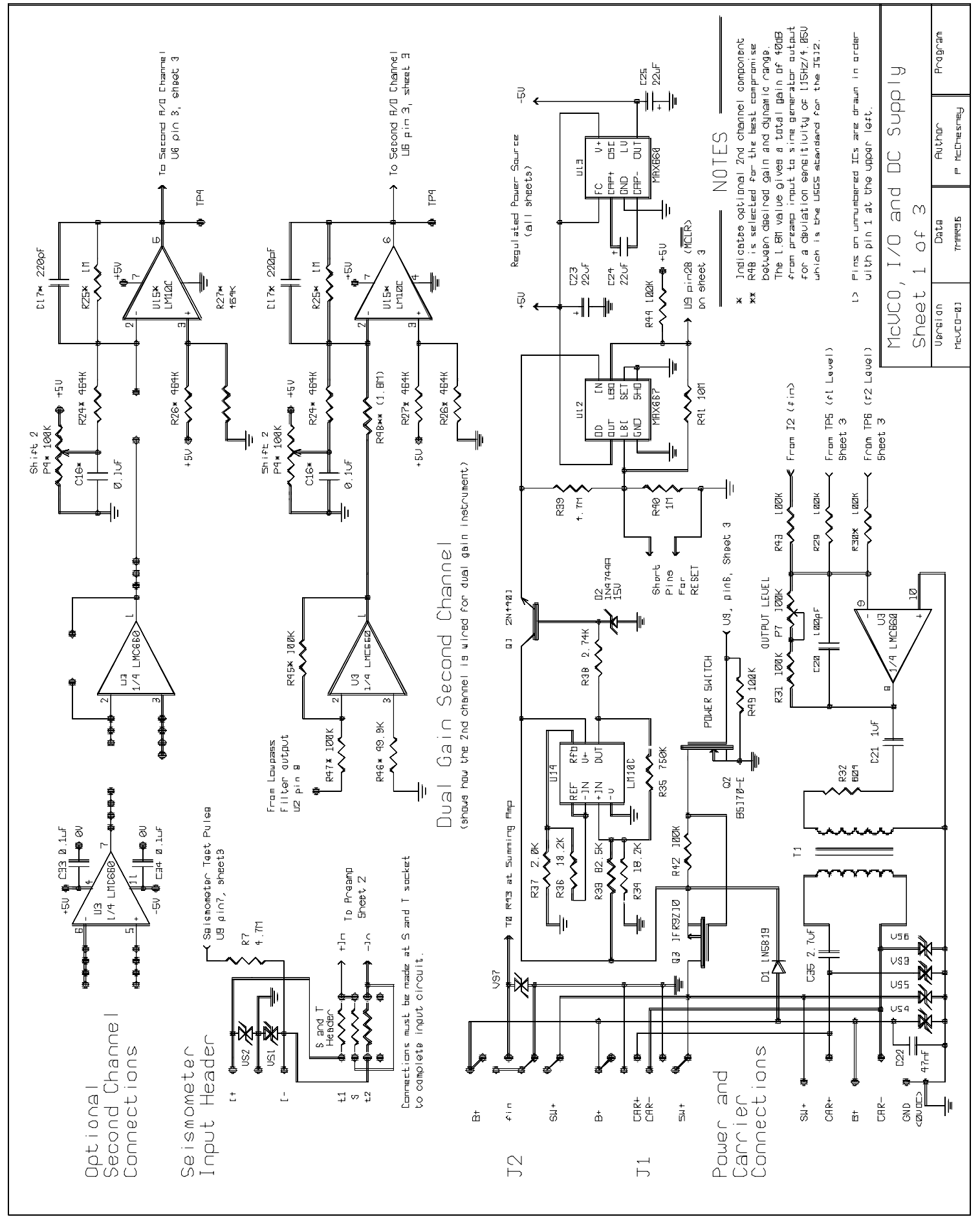

Figura 3A. I/O y corriente directa 
Parte II: Referencia Técnica

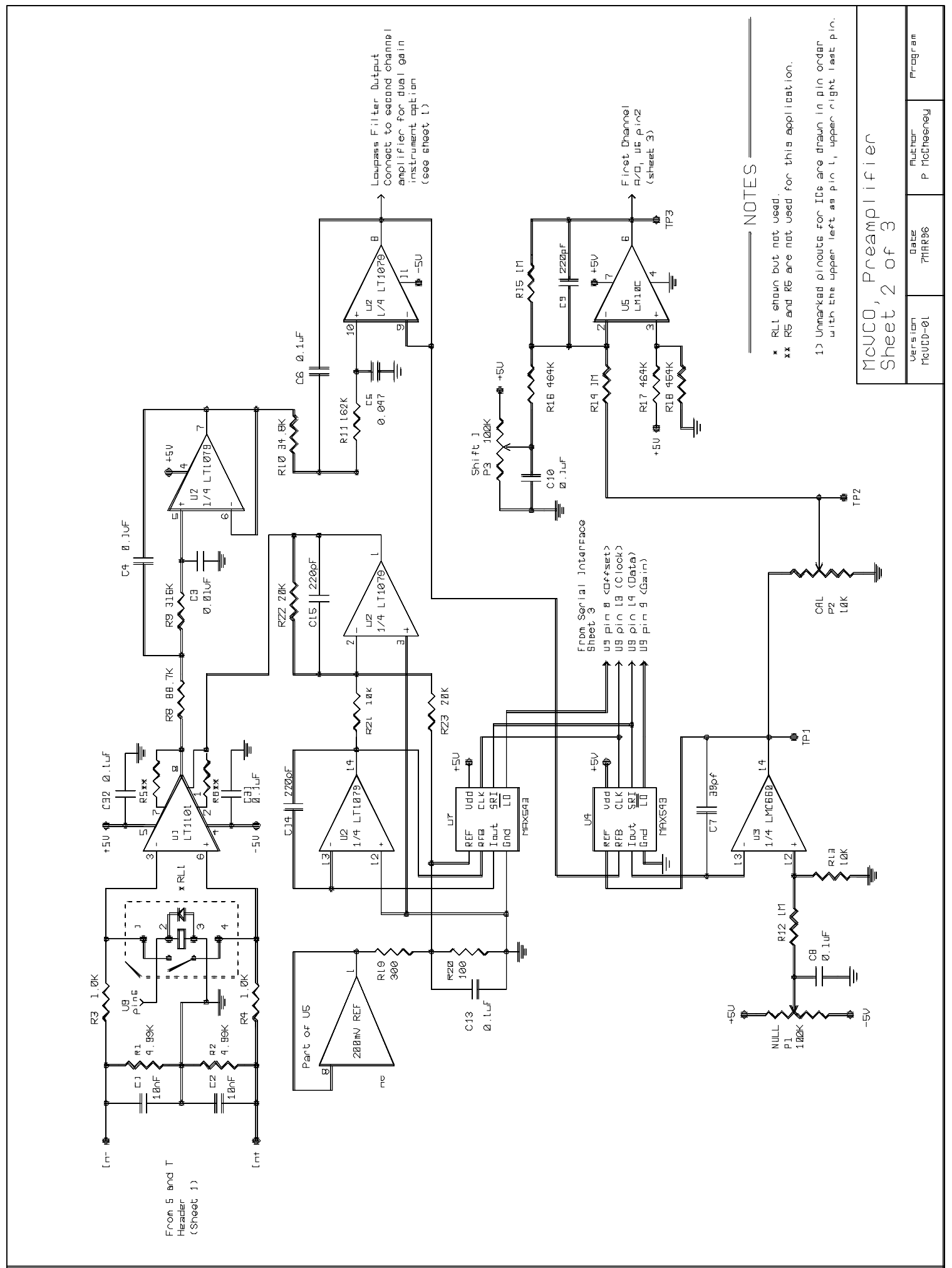

Figura 3B. Amplificador de Senal Sismica 
Parte II: Referencia Técnica

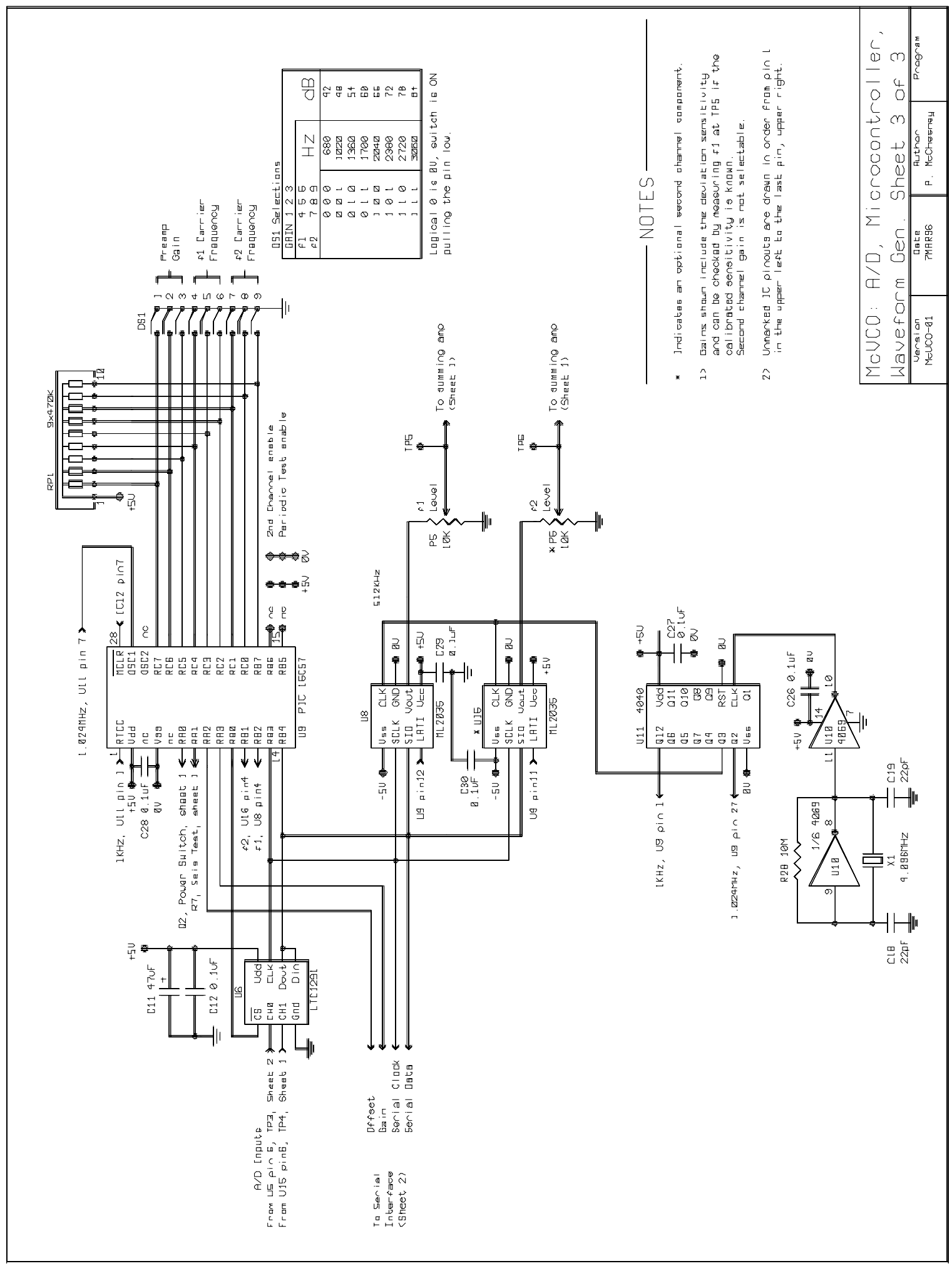

Figura 3C. ADC, Microcontrolador, y generador de ondas senoidales 


\section{Especificaciones del McVCO}

Estas especificaciones, cuando no son inherentes al diseño, son "típicas" del funcionamiento del instrumento. Intentan caracterizar al instrumento a pesar de una limitada capacidad de prueba. Los comentarios por parte de los usuarios serán bienvenidos y en caso de que haya errores en estas especificaciones, se harán las revisiones correspondientes.

\section{Amplificador de la señal sísmica}

Tipo de Entrada

Ajuste de Ganancia

Precisión de Ganancia

Respuesta en Frecuencia

Nivel de ruido de salida

Sensibilidad

Amplificador del Segundo Canal
Diferencial, con $Z_{\text {in }}=10 \mathrm{~K} \Omega$ para $\mathrm{f}<30 \mathrm{~Hz}$.

Se suministran las conexiones para las resistencias $\mathrm{S}$ y $\mathrm{T}$.

$42 \mathrm{~dB}$ en 8 pasos de $6 \mathrm{~dB}$ cada uno seleccionados por interruptor. Comúnmente el comienzo es a $42 \mathrm{~dB}$ con un máximo a $84 \mathrm{~dB}$.

Mejor del $3 \%$ en todas los rangos.

Filtro Butterworth pasa bajas de $30 \mathrm{~Hz}$ y 4 polos $(-24 \mathrm{~dB}$ /octava arriba de $30 \mathrm{~Hz}$ ). El amplificador está acoplado en forma directa, pero el rendimiento en CD es afectado por el control del offset. La atenuación por el circuito de control de offsets es menor a $3 \%$ para señales de entrada con velocidad de respuesta (slew rate) mayores a $50 \mathrm{nV} / \mathrm{seg}$ y menor a $1 \%$, para velocidad de respuesta mayores a $100 \mathrm{nV} / \mathrm{seg}$.

Ver información en Parte III, Respuesta en Frecuencia y Ganancia.

$\mathrm{Vn}($ out $)=3 \mathrm{mV}$ rms a máxima ganancia, decreciendo proporcionalmente con la ganancia. Equivalente a $20 \mathrm{mV}$ p-p en la entrada del CAD o $0.4 \%$ del rango del CAD para \pm 8 conteos.

$2.6 \mu \mathrm{V}$ rms a la entrada con máxima ganancia producen $35 \mathrm{mV}$ rms en el CAD (100 mV p-p) con $3 \mathrm{mV}$ rms de ruido para Signal to Noise Ratio (nivel de señal a ruido) de $21.3 \mathrm{~dB}$ cuando las tablas de ganancia están puestas a una sensibilidad de desviación de 41.67 $\mathrm{Hz} / \mathrm{V}$. Otras sensibilidades de desviación son similares. Ver información en Parte III, Respuesta de Frecuencia y Ganancia.

Definido por el usuario. La ganancia es de $40 \mathrm{~dB}$ para versiones del USGS con ganancia dual. 
Parte II: Referencia Técnica

\section{$\underline{\text { Modulador }}$}

Rango de Salida

Sensibilidad de desviación

Conversión Analógica a Digital (CAD)

Resolución de frecuencia

Frecuencias Centrales

Estabilidad de Frecuencia

Tipo de Salida

Amplificador de Salida

Nivel de Salida

Ruido + Distorsión $\pm 125 \mathrm{~Hz}$ de la frecuencia central, absolutamente limitada ya que la frecuencia instantánea se calcula a partir de la conversión analógica-digital (CAD) que abarca exactamente este rango.

$($ Rango de Salida $) /($ Rango del CAD $)=(250 \mathrm{~Hz}) /(5 \mathrm{~V})$ Otras sensibilidades se emulan escalando la ganancia en el amplificador de señal sísmica. Ver información en Parte III, Respuesta en Frecuencia y Ganancia.

Conversor CAD de aproximación sucesiva de 12 bits con un muestreo de 660 muestras por segundo para una operación de un canal y de 330 muestras por segundo para dos canales.

La variación mínima de frecuencia del generador es de 250 Hz/4096 y resuelve completamente el conversor CAD de 12 bits.

\section{Salida de la Portadora de Audio}

Cualquiera de las siguientes frecuencias centrales son seleccionables mediante interruptor para ambos canales.

$680,1020,1360,1700,2040,2380,2720,3060 \mathrm{~Hz}$

La salida del generador de ondas senoidales es derivada del cristal por lo que el factor más importante es el offset en la salida del amplificador de señales sísmicas. Este offset es regulado activamente por el microcontrolador para que la frecuencia de salida quede dentro de $\pm 2 \mathrm{~Hz}$ de la frecuencia central después del ajuste automático a cero en el reset y se ajuste hasta un $1 \mathrm{~Hz}$ en promedio en un periodo largo .

Acoplado por transformador con un capacitor de bloqueo de CD. El secundario está en flotación y tiene una impedancia reflejada de salida diferencial de $600 \Omega$.

El amplificador de salida es un inversor sumador con opción para una portadora de un segundo canal y la entrada de una portadora externa.

0 a $4 \mathrm{~V}$ p-p en $600 \Omega(2.6 \mathrm{dBm} \max )$.

Superior a $45 \mathrm{~dB}$ relativo a la portadora a máxima salida con una carga de $600 \Omega$. Superior a $47 \mathrm{~dB}$ relativo a la portadora en $1 \mathrm{~V} \mathrm{p}$-p con carga de $10 \mathrm{~K} \Omega$. 


\section{Energía y Medio Ambiente}

Rango de Alimentación $\quad 11$ a 20 VCD. Puede mantener la salida sin carga de un panel solar de $30 \mathrm{~W}$ y $12 \mathrm{~V}$ indefinidamente (limitada a aproximadamente 20 $\mathrm{V}$ regulada a la entrada por un supresor de transitorios de voltaje (SVT)). Un interruptor de alimentación interno asegura el reset del microcontrolador, con encendido a 11.5 volt y apagado a 10.5 volt

Consumo de Energía

Un solo canal, $220 \mathrm{~mW}$ a $12 \mathrm{VDC}(18 \mathrm{~mA})$

Dos canales, $300 \mathrm{~mW}$ a $12 \mathrm{VDC}(25 \mathrm{~mA})$.

Protección

Todas las entradas y salidas están protegidas por supresores de transitorios de voltaje de $15 \mathrm{~V}$ (SVT), a excepción del supresor de la entrada de potencia fijado en $20 \mathrm{~V}$.

El suministro de alimentación está protegido contra inversión de polaridad por una diodo en serie.

Rango de Operación por Temperatura

Las unidades con componentes de calidad industrial han sido probadas exitosamente a $-40^{\circ} \mathrm{C}$. No se han realizado pruebas de temperatura mas altas. El rango sugerido es de $-30 \mathrm{a}+50^{\circ} \mathrm{C}$ basado en la temperatura de operación de componentes de calidad industrial.

Tamaño de la Tarjeta Longitud=7.7", ancho=3.9", altura=1.0" ( $\max )$ 
Parte III: Notas de Aplicación

\section{Parte III: Notas de Aplicación}




\section{Respuesta en Frecuencia y Ganancia}

Cuando se diseñó el McVCO, se esperaba que fuera posible decir que era una imitación digital de un VCO en particular y poder copiar su respuesta. La situación no fue tan sencilla. No sólo variaban los VCO, sino que los usuarios los modificaban frecuentemente. En consecuencia, el McVCO tiene sus propias características de respuesta y diseño, y en lugar de imitar otro instrumento, trata de utilizar la tecnología de un microcontrolador para su mejor provecho. Desafortunadamente esto requiere que el usuario aprenda algo sobre cómo un McVCO imita a un VCO analógico. Debido a que no todos desean ver los detalles, se proporciona un breve resumen de las respuestas de ganancia y frecuencia seguido de una discusión más detallada.

\section{Respuesta en Ganancia}

La ganancia del McVCO se relaciona con la señal en la entrada del amplificador de señales sísmicas. No incluye ningún término de atenuación de la señal sísmica que pueda ocurrir en las resistencias $\mathrm{S}$ y T entre el sismómetro y el amplificador. El usuario decidirá sobre el amortiguamiento y la calibración del sismómetro. La impedancia de entrada que presenta el amplificador de señales sísmicas a la red $\mathrm{S}$ y $\mathrm{T}$ es de $10 \mathrm{~K} \Omega$ y debe estar incluida en los cálculos de S y T.

La ganancia electrónica del amplificador de señales sísmicas se ajusta para ofrecer una sensibilidad de desviación efectiva tal que:

$$
\Delta f=\left(S_{\text {en }}\right) \times\left(A_{\text {DIP }}\right) \times(E D S)
$$

donde: $\quad \Delta \mathrm{f}$ es el cambio en la frecuencia de salida $(\mathrm{Hz})$.

$\mathrm{S}_{\mathrm{en}}$ es la señal de entrada del amplificador de señales sísmicas (V).

$\mathrm{A}_{\mathrm{DIP}}$ es la ganancia seleccionada por el interruptor DIP $(\mathrm{V} / \mathrm{V})$.

EDS es la sensibilidad de desviación efectiva $(\mathrm{Hz} / \mathrm{V})$.

La sensibilidad de desviación efectiva es la sensibilidad de desviación calibrada especificada por el usuario para un sistema en particular. El ajuste el potenciómetro de calibración (Cal pot) para otras sensibilidades de desviación sacrificará rango dinámico debido al recorte en el CAD o en el amplificador de señales sísmicas.

\section{Discusión sobre la Respuesta de la Ganancia}

El tema de una sensibilidad de desviación efectiva surge porque la desviación real de sensibilidad del instrumento es fijada por el rango de entrada de 0 a $5 \mathrm{~V}$ del CAD y el ancho de banda del canal de $250 \mathrm{~Hz}$. Una desviación de esta sensibilidad de $50 \mathrm{~Hz} /$ volt solo se puede realizar ajustando la ganancia antes del CAD.

El McVCO tiene una ganancia programable y la utiliza para imitar otras sensibilidades de 
desviación. Cuando se programa el microcontrolador, sus tablas internas de ganancia son seleccionadas para una sensibilidad de desviación calibrada en particular. Es posible ajustar Cal, $\mathrm{P} 2$, el potenciómetro de calibración para otras calibraciones, pero hacer esto disminuirá el rango dinámico del amplificador de señales sísmicas porque el potenciómetro de calibración proporciona una atenuación desde la salida de $10 \mathrm{~V}$ p-p del amplificador de ganancia programable hacia la entrada de $5 \mathrm{~V}$ p-p del CAD.

El máximo rango dinámico se logra cuando la salida de $10 \mathrm{~V}$ p-p varía en toda su rango al mismo tiempo que la entrada del CAD hace lo mismo. Si se tiene en cuenta que los amplificadores involucrados no alcanzan los valores fijos por la alimentación y para algún offset, la ganancia del potenciómetro de calibración Cal, $\mathrm{P} 2$, para un rango dinámico máximo será:

$$
\mathrm{V}_{\text {salida }} / \mathrm{v}_{\text {entrada }}=4.9 \mathrm{v} / 9.5 \mathrm{v}=0.5158
$$

Este es el punto óptimo del potenciómetro de calibración, Cal, P2, punto en el que se encuentra la calibración y el rango dinámico ideal. El potenciómetro se proporciona para corregir errores de ganancia en el amplificador de señales sísmicas mientras se mantiene el rango dinámico completo y no como un medio para cambiar la calibración.

Suponga que una recalibración sea necesaria. Como un ejemplo, la "Pacific Northwest Seismographic Network,PNSN) (Red Sismográfica del Noroeste del Pacífico) utiliza una desviación calibrada de $41.67 \mathrm{~Hz} /$ volt y un valor común del USGS es de $28.40 \mathrm{~Hz} /$ volt. ¿Cuánto margen dinámico se pierde recalibrando el instrumento del PNSN con el potenciómetro de calibración en comparación a uno del USGS?

Cuando se cambia la calibración, la ganancia del potenciómetro de calibración Cal, P2, se reduce a 0.5158 a 0.3515 . Esto significa que una salida del rango total del amplificador de ganancia programable produce $0.3115 \times 9.5 \mathrm{~V}$ p-p en la entrada del CAD o sea $3.34 \mathrm{~V}$ p-p en lugar de la amplitud total de $5 \mathrm{~V}$ p-p. La pérdida en el rango dinámico es de $20 \log (3.34 / 5)=-3.5 \mathrm{~dB}$. Esto puede no ser tan grave, pero la mejor forma de recalibrar es instalar un microcontrolador con las tablas de ganancias correctas si se anticipan señales fuertes. Vea la Introducción como un recurso de microcontroladores programados.

Si se desean grandes cambios en la ganancia, se pueden substraer $20 \mathrm{~dB}$ del amplificador de instrumentación de entrada instalando puentes en las posiciones del circuito marcadas como R5 y R6 cerca de U1. Otros valores de ganancia se pueden obtener con resistencias instalados en el sitio. Vea la hoja de datos técnicos Linear Technology sobre el circuito LT1101. Incrementar la ganancia más allá de los $90 \mathrm{~dB}$ que aproximadamente puede obtenerse con el potenciómetro de calibración Cal, P2, no es aconsejable debido al ruido inherente del amplificador de entrada.

La impedancia de entrada de $10 \mathrm{~K} \Omega$ del McVCO se debe a la combinación en serie de R1 y R2. Se puede utilizar otro par de resistencias de igual valor para modificar la impedancia. Estas deben ser resistencias de película metálica de $1 \%$ para que la razón de rechazo de modo común sea preservada y el ruido minimizado. 
Existe un segundo canal en el McVCO para aquellos que deseen realizar sus propias interconexiones de sensores. Vea "Utilización del Segundo Canal de Ganancia Baja" en la Parte III de este manual.

\section{Respuesta en Frecuencia}

La atenuación en la frecuencia superior del McVCO se determina a partir de un filtro activo Butterworth de 4 polos. El punto de $-3 \mathrm{~dB}$ del filtro es a $30 \mathrm{~Hz}$ y la atenuación se incrementa en $24 \mathrm{~dB} /$ octava.

No hay corte de frecuencia inferior. A una cierta amplitud y frecuencia el lazo de retroalimentación que produce la corrección de offset para el amplificador de señales sísmicas comenzará a atenuar y a distorsionar una señal de período muy largo. El tamaño de esta corrección está por debajo del nivel de ruido del amplificador de entrada y se lleva a cabo en intervalos de 32 segundos. En consecuencia, su efecto en las señales con períodos menores a 32 seg es indetectable. El sistema de corrección de offsets evita efectivamente que el amplificador de señales sísmicas funcione como un amplificador de $\mathrm{CD}$, a pesar de que esté acoplado en forma directa.

\section{Discusión sobre la Respuesta en Frecuencia}

Aunque el McVCO es analógico en su entrada y en su salida, en su núcleo es un sistema digital de datos muestreados. En el modo de dos canales, la velocidad de muestro es de 330 muestras por segundo y en modo de un canal, apenas el doble de esto. Esto le da dos trabajos al filtro pasa bajas. Debe reducir el ruido limitando el ancho de banda a las frecuencias de interés y evitar el ruido más allá de la mitad de la velocidad de muestreo del aliasing en la banda de paso. La atenuación del ruido a la mitad de la velocidad de muestreo para el modo de dos canales es aproximadamente de $60 \mathrm{~dB}$ y para el modo de un canal, es superior a $80 \mathrm{~dB}$. La respuesta de fase del filtro Butterworth en la banda de frecuencias no es lineal pero es estándar y es descrito ampliamente en la literatura.

Lo que ocurre con la respuesta del McVCO en el extremo inferior de su respuesta en frecuencia es mucho menos convencional. No hay filtro pasa altas y el amplificador de señales sísmicas es de acoplamiento directo. Sin embargo, las señales de períodos muy largos son distorsionadas y atenuadas por el circuito de corrección de offset.

El principio detrás del sistema de corrección de offset es sencillo. Las señales sísmicas después de un tiempo tienen un valor promedio de cero no así los voltajes de offset. En consecuencia, es posible cancelar los offsets y preservar la señal ajustando cuidadosamente el voltaje a través de un 
lazo de realimentación para mantener un valor promedio de cero en la entrada del CAD.

El McVCO lleva a cabo este proceso manteniendo una cuenta de offset de 12 bits en el CDA, U7. Esta cuenta produce un voltaje que se suma a la señal en la salida de U1, la primera etapa del amplificador de señales sísmicas. La polaridad del voltaje sumado a U1 es la requerida para cancelar el voltaje en la entrada del CAD. Cada 32 segundos el microcontrolador observa la conversión analógica-digital más reciente e incrementa o decrementa la cuenta del offset para que la entrada analógica-digital regrese hacia cero (el centro de la amplitud de entrada del CAD es de aproximadamente $2.5 \mathrm{VCD}$ ).

Los componentes del voltaje a la entrada del CAD son: la señal sísmica, el ruido y el offset del amplificador de las señales sísmicas. Todo ajuste particular realizado al conteo del CDA puede ser una respuesta a la señal o ruido y puede estar mal en lo que a la cancelación del offset se refiere. Sin embargo, después de muchos ciclos de corrección de offset, la señal y el ruido con valores promedio de cero durante el período de corrección de 32 seg desaparecerá de la cuenta del CDA. La cuenta del CDA contendrá el offset y una fracción de todos los componentes de señales con períodos mayores a $32 \mathrm{seg}$. Es este offset y parte de la señal de período largo lo que se remueve de la señal de entrada en la salida de U1.

Aunque el circuito de corrección de offset atenúa las señales con períodos mayores a $32 \mathrm{seg}$, el $\mathrm{McVCO}$ puede exitosamente medir señales con períodos mayores a éste. Sin embargo, antes de hablar sobre estas señales de períodos mayores, es mejor finalizar el tema sobre las señales con períodos menores al periodo de corrección de offset.

Las señales con períodos menores a 32 seg son sujetos a lo más por una corrección cada 32 seg. El tamaño de cualquier corrección es \pm 1 cuenta del CDA. El rango del voltaje de salida del circuito de corrección es de $\pm 50 \mathrm{mV}$. Dado que existen un total de 4096 cuentas del CDA, el cambio de una cuenta es un cambio de $24.4 \mu \mathrm{V}$. Esta voltaje correspondiente a una cuenta simple no es amplificada por la ganancia de $40 \mathrm{~dB}$ de la etapa de entrada y por lo tanto corresponde a $0.244 \mu \mathrm{V}$ a la entrada a U1, un circuito LT1101. El fabricante, Linear Technology, especifica que el ruido típico para señales de frecuencias de 0.1 a $10 \mathrm{~Hz}$ del LT1101 es $0.9 \mu \mathrm{V}$ p-p. Un cambio de una cuenta simple es menor que $1 / 3$ de este nivel de ruido y es virtualmente invisible para señales con períodos menores que el período de actualización de 32 seg de la cuenta de offset.

Para las señales con períodos mayores a 32 seg la situación es más complicada. La pregunta es: ¿Cuántas correcciones de offset puede tolerar una señal antes de que ya no se pueda utilizar más? El cambio en la señal depende tanto del período como de la amplitud de la señal. Las señales grandes cambiarán proporcionalmente menos que las pequeñas y las señales de periodo más largo tendrán más correcciones de offset durante cada ciclo que las señales de periodo más corto. Este producto de la amplitud y la frecuencia describe la velocidad de respuesta (slew rate $\mathbf{S}$ ) de una onda senoidal dada por la siguiente expresión, donde $\mathbf{A}$ es la amplitud de pico a pico y $\mathbf{P}$ es el período:

$$
\mathbf{S}=\mathbf{A}_{(\mathbf{p}-\mathbf{p})} \times \pi / \mathbf{P}
$$


La función del lazo de realimentación en la corrección del offset es siempre llevar la entrada del CAD hacia el nivel de señal cero. Se opone a la señal de entrada mientras se aleja de cero y ayuda a la señal mientras se acerca a cero. Esta acción atenúa la señal de entrada y el efecto del circuito de corrección de offset sobre señales de periodo largo puede ser descrito parcialmente como una atenuación que varía con la velocidad de respuesta de la señal. La Figura 4 muestra esta relación.

La curva de la Figura 4 muestra un cambio gradual en la atenuación de las señales con velocidades de respuesta mayores a $50 \mathrm{nV} / \mathrm{seg}$. Muestra un rápido incremento de la atenuación para las señales con velocidades de respuesta menores que este valor. En consecuencia, debe prestarse atención al funcionamiento del McVCO para señales con esta velocidad de respuesta.

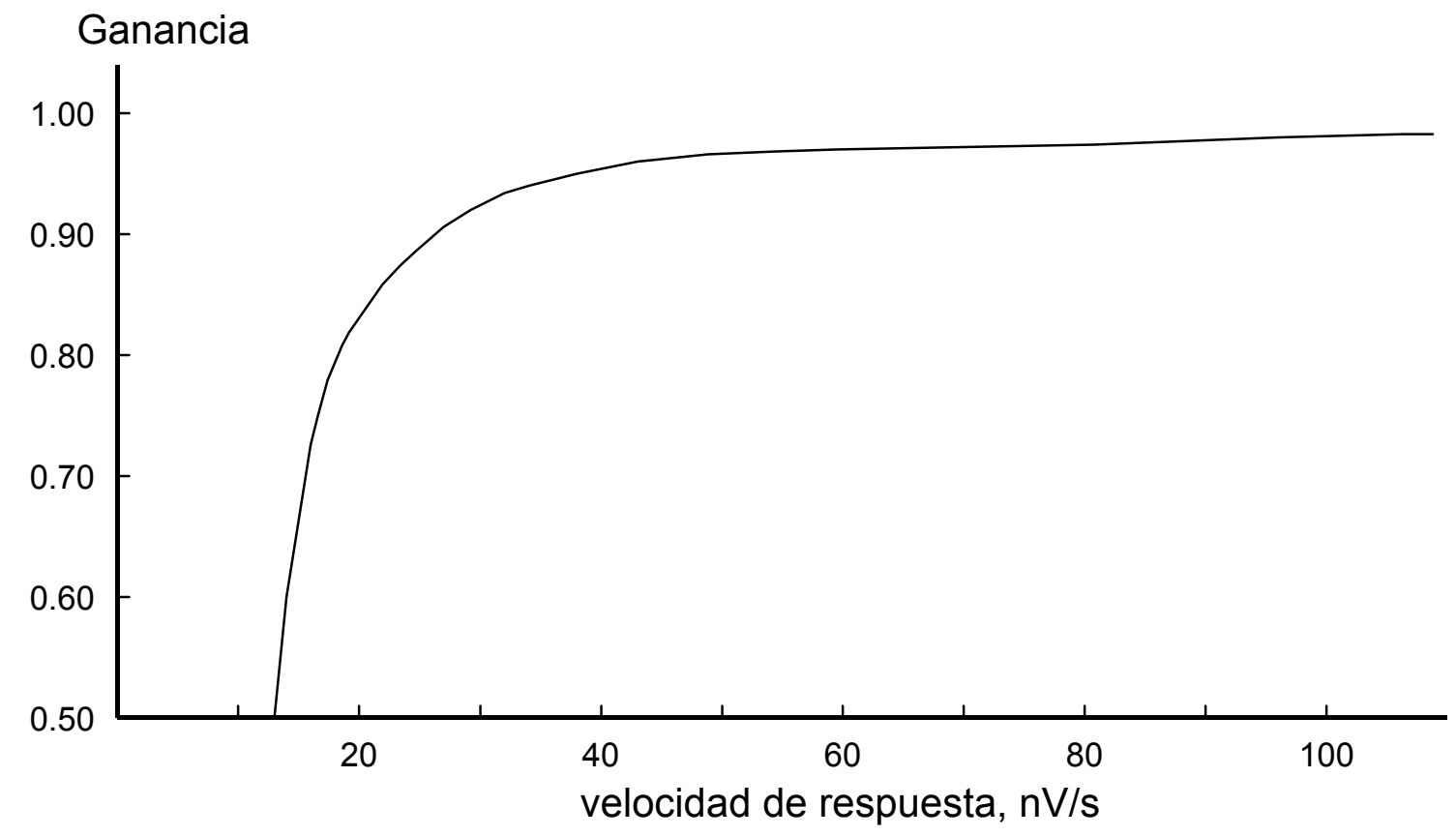

Figura 4. El efecto de la corrección de offset en la amplitud de la señal contra los cambios de la velocidad de respuesta (slew rate) de la señal varía. La atenuación se expresa como ganancia (Vout /Vin) normalizada a uno en comparación a una señal sin atenuar con una velocidad de respuesta mucho más elevada.

El McVCO está especificado para tener una relación de señal a ruido mayor que $20 \mathrm{~dB}$ en el CAD para una señal de $2.6 \mu \mathrm{V}$ rms $(7.35 \mu \mathrm{V}$ p-p) a máxima ganancia. El período de una señal con esta amplitud y una velocidad de respuesta de $50 \mathrm{nV} / \mathrm{seg}$ es de $460 \mathrm{seg}$. Si la atenuación de aproximadamente $3 \%$ a $50 \mathrm{nV} / \mathrm{seg}$ es aceptable, entonces ésta puede ser tomada como la amplitud más pequeña y la señal del período más largo que el McVCO puede medir sin interferencia del circuito de corrección de offset. Si señales mayores con velocidad de respuestas más rápidas son de interés, entonces el $\mathrm{McVCO}$ puede medir señales con períodos mayores que $460 \mathrm{seg}$. 
La atenuación no es el único efecto del circuito de corrección de offsets en la señal. A medida que la velocidad de respuesta decrece a $25 \mathrm{nV} / \mathrm{seg}$, la distorsión se torna claramente visible en la señal del CAD. La distorsión en la señal proviene de la forma de onda de corrección del offset. Es una rampa positiva o negativa. Durante el semiciclo positivo de la señal en el CAD, el circuito de corrección varía negativamente una cuenta cada $32 \mathrm{seg}$, mientras que para el semiciclo negativo varía positivamente a la misma tasa. Esta rampa se suma a la señal de entrada en la salida de U1. Cuando la velocidad de respuesta decrece, la rampa se amplía y la distorsión aumenta. La Figura 5 muestra la distorsión de una señal con una velocidad de respuesta de $16 \mathrm{nV} / \mathrm{seg}$. Esta señal tiene una ganancia normalizada de aproximadamente 0.7 . 


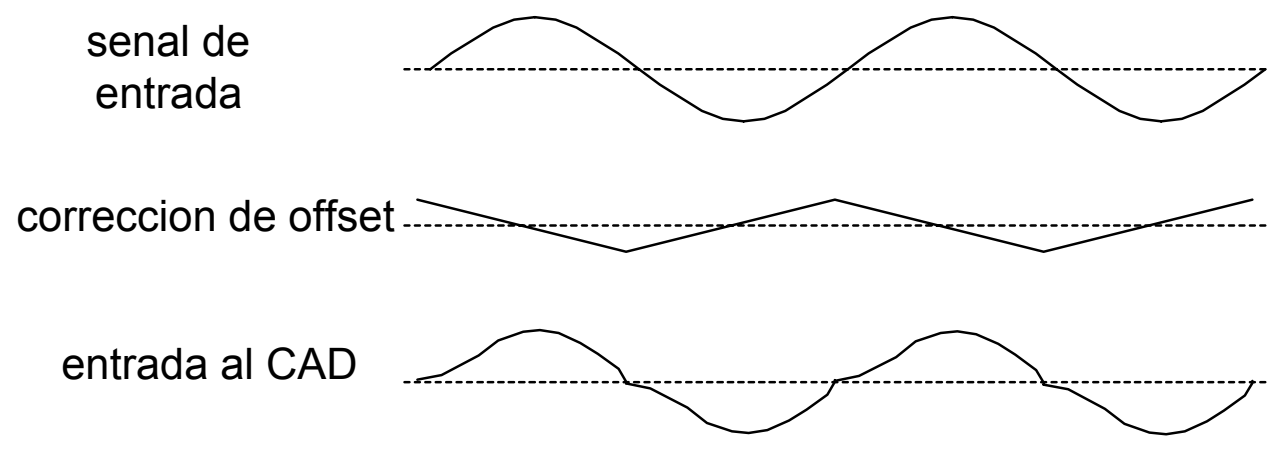

Figura 5. La distorsión de una señal de $16 \mathrm{nV} / \mathrm{seg}$ provocada por el circuito de corrección de offset. El dibujo no está en escala. La señal del CAD es 0.7 veces la amplitud de una señal no atenuada con una velocidad de respuesta más rápida.

Aunque el circuito de corrección de offset produce una respuesta de frecuencia baja no convencional, el McVCO puede eficazmente medir las señales de períodos largos si tienen velocidades de respuesta mayores a $50 \mathrm{nV} / \mathrm{seg}$. Si se debe trabajar hasta $\mathrm{CD}$, el segundo canal del McVCO se encuentra fuera del lazo de corrección. Vea la Parte III, Utilización del Segundo Canal de Ganancia Baja. 


\section{Utilización del Segundo Canal}

La mayor parte de los McVCO que se han proporcionado están configurados para una operación de ganancia dual en la que un primer canal de alta ganancia se complementa con un segundo canal de baja ganancia. El segundo canal está conectado a la salida del filtro pasa bajas del primer canal y comparte la misma señal. El propósito de esta configuración es el de incrementar el rango dinámico del instrumento en presencia de fuertes señales de entrada. Existe la posibilidad de que los usuarios de este instrumento deseen cambiar este modo de operación por dos motivos. Puede no haber espacio en la red de telemetría para un segundo canal, o el segundo canal puede requerirse para otro propósito. Cómo anular el segundo canal y cómo interconectar otras señales se explicará a continuación.

\section{Cómo Deshabilitar el Segundo Canal}

La forma más fácil de deshabilitar el segundo canal es retirando el generador de ondas senoidales U16 del circuito y aterrizando la entrada del segundo canal al amplificador sumador de salida girando el control de nivel f2, P6 completamente en sentido contrario a las manecillas del reloj. Este método crudo tiene la ventaja de ahorrar alrededor de $7 \mathrm{~mA}$ y evita que el segundo canal emita señales o ruidos no deseados a la red de telemetría. El generador de onda senoidal ML2035 toma $2 \mathrm{~mA}$ en el modo de bajo consumo controlado por el microcontrolador. Así que extrayendo el circuito ahorrará más energía que apagándolo con el microcontrolador. Se pueden ahorrar adicionalmente $0.5 \mathrm{~mA}$ extrayendo el amplificador de desvío de nivel del segundo canal, U15.

Hay una forma de indicarle al microcontrolador que se desea el modo de un canal. Si el pin 16 de U9 del microcontrolador se mantiene bajo, el controlador no pedirá una muestra del segundo canal del CAD. Esto duplicará la velocidad de muestreo para el primer canal. El aumento de la velocidad de muestreo incrementa la efectividad de la función anti-aliasing del filtro pasa bajas del primer canal. La atenuación de este filtro a la mitad de la velocidad de muestreo durante una operación de dos canales es de $60 \mathrm{~dB}$. Esto aumenta a $80 \mathrm{~dB}$ para una operación de un canal.

La atenuación incrementada es una ventaja si existen fuentes de ruido con componentes de frecuencia por encima de la mitad de la velocidad de muestreo. La velocidad de muestreo para una operación de dos canales es de 330 pruebas / seg. La velocidad para un canal es de 660 muestra / seg. Aumentando el techo del ruido superficial con el ruido por aliasing reduce la capacidad del instrumento para transmitir pequeñas señales, aunque otras fuentes de ruido en el enlace de telemetría son por lo general más importantes para la reducción de la sensibilidad total que el cambio de operación de uno o dos canales.

Si es necesario operar sólo el segundo canal, entonces el primer canal puede deshabilitarse en forma similar si se extraen U8 y U5. No existe ninguna manera de decirle al procesador que tome muestras sólo del segundo canal, por lo que el pin 16 de U9 debe mantenerse alto $(5 \mathrm{~V})$ para todas las mediciones del segundo canal excepto para aquellas realizadas durante el ciclo de prueba. (Vea la Parte III, Control del voltaje de la Batería). 


\section{Interconexión del Segundo Canal}

Esta es una guía preliminar para la utilización del segundo canal, destinada a aquellos usuarios con cierta experiencia en el diseño de circuitos analógicos. No se pueden cubrir aplicaciones específicas, por lo que el enfoque será en las características generales del segundo canal.

El diagrama esquemático (Figura 3A) muestra el segundo canal de dos formas. La mitad de la página muestra un segundo canal de baja ganancia conectado a la salida del filtro pasa bajas del primer canal. Encima del mismo, algunos de los mismos componentes se muestran desconectados. Estos componentes están disponibles para que el usuario realice la conexión del segundo canal y para este fin, se dispone de una pequeña área cerca de U3 para implementar circuitos prototipo.

Los dos amplificadores operacionales no usados de U3 pueden o no adaptarse a una aplicación particular. El amplificador cuádruple LMC660 no es un amplificador de CD de precisión, y probablemente no sea el adecuado para circuitos de CD de ganancia alta. Fue escogido porque su rango de salida de extremo a extremo lo hizo útil en el circuito del amplificador de ganancia variable mientras que el producto ganancia - ancho de banda de $1.1 \mathrm{MHz}$, la baja distorsión y la capacidad de manejar $600 \Omega$ lo hacen una buena elección para el amplificador sumador de salida. Encontrar estas características en un amplificador de baja potencia es difícil. No se recomienda la sustitución del LMC660 en U3 por otro amplificador operacional cuádruple. Consulte la hoja de información de LMC660 para ver sus especificaciones completas.

Independientemente de que se utilicen o no los amplificadores opcionales de la tarjeta, el lugar de entrada al segundo canal es a través del circuito nivelador de amplitud U15. Este circuito desplaza las señales al centro de $2.5 \mathrm{VCD}$ del rango 0 a $5 \mathrm{~V}$ del conversor CAD. El circuito es un inversor sumador polarizado a $2.5 \mathrm{VCD}$ en la entrada no inversora. El punto de suma recibe entrada desde P4 y la señal a través de resistencias para selección de ganancia. A estas resistencias se eligen para que con la señal de entrada máxima, P4 pueda ajustarse para una variación completa alrededor del centro de la señal de entrada al CAD.

A diferencia de un VCO convencional diseñado con componentes analógicos, el McVCO posee una sensibilidad del modulador fija. El rango de entrada de $5 \mathrm{~V}$ del convertidor CAD corresponde exactamente a una desviación de $\pm 125 \mathrm{~Hz}$ de la frecuencia central para una sensibilidad constante de modulación de $50 \mathrm{~Hz} / \mathrm{V}$. Con frecuencia se requiere operar con una sensibilidad de desviación diferente a esta sensibilidad del modulador. Esta puede lograrse cambiando la ganancia antes del modulador para que éste responda a una señal de entrada en el amplificador de señales sísmicas como si tuviera una sensibilidad de desviación diferente.

Desafortunadamente esto puede ser confuso. Una vez que la sensibilidad del modulador y la sensibilidad de desviación no son las mismas, el ajuste de la ganancia calibrada del instrumento difiere de la ganancia actual de la señal. Supongamos que se desea operar el segundo canal del McVCO a una ganancia calibrada de $100(40 \mathrm{~dB})$ en un sistema que está establecido para una sensibilidad de desviación de $115 \mathrm{~Hz} / 4.05 \mathrm{~V}$. La sensibilidad del modulador $(50 \mathrm{~Hz} / \mathrm{V})$ del 
McVCO es muy grande y por tanto la ganancia del amplificador de señales sísmicas debe ser reducida proporcionalmente.

La ganancia de la señal desde el amplificador $\left(\mathbf{A}_{\text {signal }}\right)$ es

$$
\mathbf{A}_{\text {signal }}=\mathbf{A}_{\text {cal }} \times \mathbf{D}_{\text {sens }} / \mathbf{M}_{\text {sens }}
$$

donde

$$
\begin{aligned}
& \mathbf{A}_{\text {cal }} \text { es la ganancia calibrada deseada }(\mathrm{V} / \mathrm{V}) \text {. } \\
& \mathbf{D}_{\text {sens }} \text { es la sensibilidad de desviación del sistema }(\mathrm{Hz} / \mathrm{V}) \text {. } \\
& \mathbf{M}_{\text {sens }} \text { es la sensibilidad del modulador del } \operatorname{McVCO}(50 \mathrm{~Hz} / \mathrm{V}) \text {. }
\end{aligned}
$$

En este caso, la ganancia de la señal del amplificador de señales sísmicas debe estar ajustada a 56.79 para que el McVCO imite a un instrumento con la sensibilidad de desviación requerida establecida para una ganancia de 100. Cada vez que el McVCO sea utilizado en un sistema con una sensibilidad de desviación que difiere de la de su modulador, la ganancia de la señal del amplificador debe ser escalada para compensar. Ver Parte III, "Respuesta de Frecuencia y Ganancia del McVCO" para más información.

Cuando se realiza la conexión de una señal al segundo canal es importante tener en cuenta que el McVCO es un sistema de datos muestreados y no puede procesar señales por encima de la mitad de la velocidad de muestreo. Las señales y los ruidos por encima de la mitad de la velocidad de muestreo aparecerán como ruidos de aliasing e interferirán con las señales en la banda de paso. Generalmente se utiliza un filtro pasa bajas para controlar este ruido de aliasing y el usuario se confronta con el problema de diseñar el filtro.

Para comenzar, la velocidad de muestreo del McVCO con el segundo canal operando es de 330 muestras/seg, por lo que la atenuación del filtro a $165 \mathrm{~Hz}$ es de consideración. Al decidir sobre cuál debe ser esta atenuación, deben considerarse varios aspectos. Ante todo, ¿es necesaria? Si la entrada no tiene componentes de frecuencia por encima de $165 \mathrm{~Hz}$ o una medición de baja resolución es suficiente, entonces no se requiere ningún filtrado.

Por otra parte, si se requieren mediciones de gran sensibilidad en presencia de ruido, entonces el filtro es importante. El máximo rango dinámico del McVCO se encuentra limitado a $72 \mathrm{~dB}$ por el CAD de 12 bits. Si la señal de salida del McVCO viaja a través de enlaces de radio o teléfono, entonces este margen se degrada y el diseño para un techo de ruido de $60 \mathrm{~dB}$ por debajo del nivel máximo de la señal es indicado. El techo de ruido contiene todos los ruidos, no sólo los ruidos por aliasing, por lo que la limitación del ancho de banda en el rango de frecuencias de interés es otro factor a considerar. Finalmente, si la información recogida a través de la red de telemetría está sujeta a un procesamiento posterior, entonces puede ser posible que la respuesta de fase del filtro deba ser considerada.

Seleccionar una respuesta de filtro es frecuentemente un compromiso complejo determinado en primer lugar por los requerimientos de la aplicación. Algunos filtros tienen una caída de atenuación más acentuados; otros tienen anchos de banda más planos y otros tienen mejores respuestas en fase. No existe el filtro perfecto y el usuario debe decidir cuáles son las características más importantes. 


\section{Ciclo de Prueba del McVCO}

Al dar un reset, o en intervalos de 24 horas, el McVCO ejecuta una rutina de prueba con el fin de darle al usuario una idea cualitativa de la condición del instrumento. Unidades con números de identificación mayores a 125 realizan estas verificaciones cada 18 horas. A continuación se da una descripción del ciclo de prueba y se sugiere cómo utilizarlo. Algunas versiones anteriores pueden no tener todos los elementos descritos. La versión realizada por la PNSN tiene un ciclo de prueba diferente.

El ciclo de prueba se divide en 3 partes. Para comenzar, un tono de $21.25 \mathrm{~Hz}$ modula la subportadora para señalar el comienzo del ciclo al sistema de colección de datos. A esto le sigue una serie de 8 pulsos de prueba del sismómetro. El ciclo finaliza con trece pulsos que representan la regulación de ganancia del instrumento y un número de identificación del mismo. Las unidades más recientes con números de identificación mayores a 125 agregan doce pulsos más cuando están en modo de un canal. Estos pulsos son el resultado de una medición del CAD del segundo canal. Por lo general, esta medición del CAD es el voltaje de la batería. Esta aplicación del segundo canal se describe en la Parte III, Control del Voltaje de la Batería.

El tiempo total del ciclo de prueba depende de cómo es iniciado. Si se inició desde un reset; entonces se requieren 10 segundos para el ajuste automático a cero seguida por 10.25 segundos del tono de $21.25 \mathrm{~Hz}$. Luego el sismómetro es verificado durante 18 segundos y los pulsos con números de identificación de ganancia llevan 13 segundos. Si el CAD del segundo canal se utiliza durante el ciclo de prueba, se necesitan 12 segundos adicionales.

Durante el ajuste automático a cero, la salida del McVCO es determinada por el procesador a la frecuencia central del canal. En consecuencia proporciona un nivel de referencia cero para discriminadores y una prueba de campo para la correcta frecuencia del canal. Los niveles de referencia también son provistos por la ganancia, el número de identificación y los pulsos del CAD del segundo canal, que son modulaciones artificiales de la subportadora en $0 \mathrm{y} \pm 62.5 \mathrm{~Hz}$ a partir de la frecuencia central. El tono de $21.25 \mathrm{~Hz}$ modula en $\pm 93.75 \mathrm{~Hz}$. Muchos discriminadores tienen filtros pasa bajas con frecuencias de corte de $20 \mathrm{~Hz} \mathrm{a}-3 \mathrm{~dB}$. Si éste es el caso, la amplitud de $21.25 \mathrm{~Hz}$ será la misma que la ganancia, el número de identificación y los pulsos del CAD del segundo canal en la salida del discriminador.

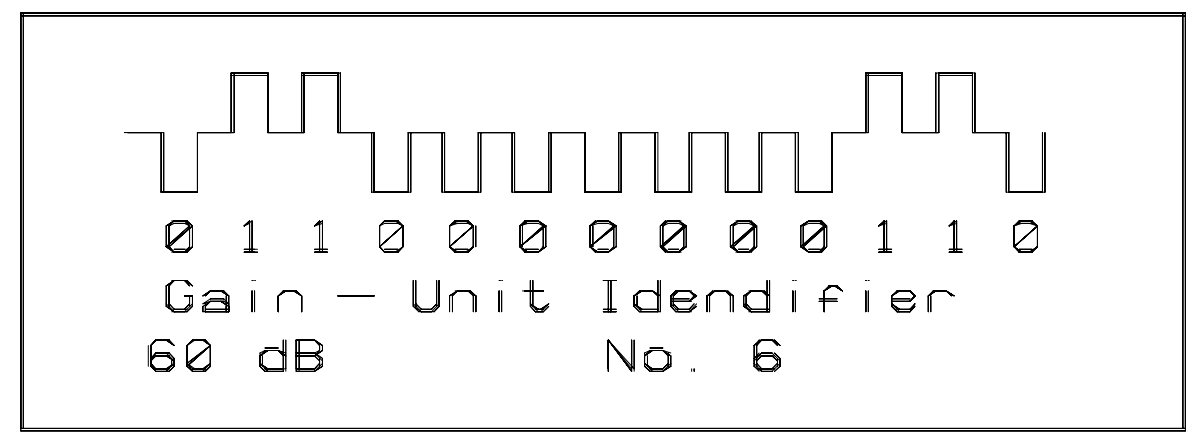

Figura 6. Ganancia demodulada y serie de pulsos del número de identificación. 
En la Figura 6, los primeros tres pulsos muestran al ajuste de ganancia del interruptor DIP del instrumento, desde el último reset de la unidad. Los últimos diez pulsos son un número binario, con el bit más significativo primero, que es el identificador de la unidad. Este número está programado en el microcontrolador. Por sí solos también muestran el estado de operación del discriminador. Cada pulso dura 0.5 segundos y está dentro del ancho de banda del discriminador. La salida demodulada debe mostrar flancos ascendentes bien definidos con un sobrepaso de aproximadamente $20 \%$. Los extremos de la onda no deben pandearse.

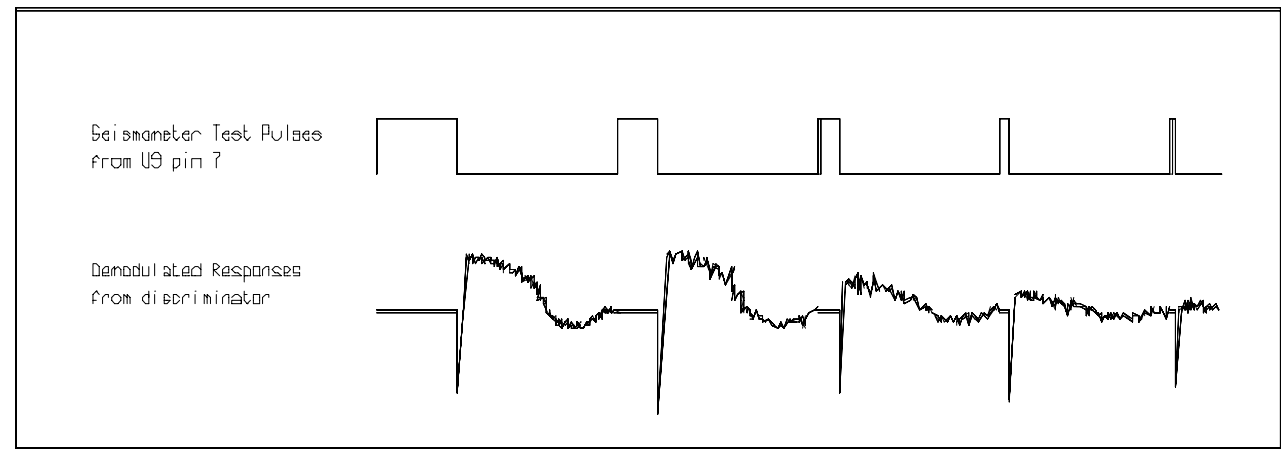

\section{Figura 7. Los pulsos de prueba del sismómetro y la ganancia típica.}

Los pulsos de prueba del sismómetro mostrados en la traza superior de la Figura 7 son señales del procesador inyectadas a la entrada del amplificador de señales sísmicas (vea la Figura $\mathbf{3 A}$ ). Cinco de los ocho pulsos pueden verse en la figura. El primer pulso dura 1 seg y es seguido 2 seg después por el segundo pulso. Cada pulso tiene la mitad de la duración del anterior y lo sigue por 2 seg. La bobina del sismómetro recibe corriente durante cada pulso y la masa del sismómetro se desplaza una distancia aproximadamente proporcional a la duración del pulso.

La traza inferior de la Figura 7 es la respuesta demodulada del instrumento a los pulsos de prueba. Mientras se aplica el primer pulso al sismómetro, el McVCO está programado para operar en la frecuencia central del canal. Cuando el pulso termina, el instrumento comienza a realizar una medición con el CAD y a modular la salida de frecuencia. Lo primero que el CAD nota es la recuperación del amplificador de señales sísmicas del pulso de prueba. Esto se muestra en la traza inferior como el pico negativo bien definido seguido por el crecimiento al pico positivo. El pico representa la respuesta amortiguada del sismómetro al pulso de prueba mas la señal sísmica y el ruido. Después de los 2 seg de medición del CAD el instrumento se ajusta nuevamente a la frecuencia central mientras que el siguiente pulso es aplicado al sismómetro. La medición comienza otra vez con la finalización del pulso de prueba y así sucesivamente para un total de ocho pulsos.

La respuesta del sismómetro a estos pulsos de prueba depende de muchas cosas que deben ser especificados con exactitud. Entre otras cosas, depende del tipo de sismómetro, de la amortiguación y atenuación S y T y el ajuste de ganancia del amplificador. Por lo general la traza inferior es una señal para la estación que debe ser registrada y comparada con registros anteriores. 
El tamaño de la respuesta no debe variar aunque el ruido y la señal sísmica sobrepuesta pudieran alterar su apariencia. Reducciones en la respuesta pueden indicar problemas con el sismómetro, como por ejemplo problemas de inclinación o de humedad. Una cable o sismómetro abierto mostrará signos del pulso de prueba sin la respuesta del sismómetro, mientras que el socket $\mathrm{S}$ y $\mathrm{T}$ abierto no mostrará ni el pulso ni la respuesta.

La rutina de prueba es especialmente útil por aquellos que pueden ver la salida demodulada del McVCO. Pero hay algunas características de dicho ciclo que se pueden observar directamente. El ajuste automático a cero hace que la unidad se detenga en la frecuencia central del canal durante $10 \mathrm{seg}$. Cada vez que sea necesario comprobar esta frecuencia, sólo deberá darse un reset mientras se observa la salida con un contador de frecuencia. La respuesta de los pulsos de prueba del sismómetro no pueden observarse directamente sin demodulación, pero el pulso mismo es útil para localizar fallas en el amplificador de las señales sísmicas. Los pulsos de prueba inyectan una señal entre 1 y $2 \mathrm{mV}$ a la entrada del amplificador que puede seguirse con un osciloscopio para verificar su operación. Para generar la señal, de un reset a la unidad y 20 segundos después aparecerán los pulsos. Si se tiene disponible un amplificador de audiofrecuencia o un escáner, el tono $21.25 \mathrm{~Hz}$ y los pulsos de ganancia y número de identificación podrán ser escuchados. Dichos pulsos son lo suficientemente grandes y lentos para que puedan interpretarse con sólo escuchar o mirar la portadora modulada con un osciloscopio. 


\section{Monitoreo del Voltaje de la Batería}

Los McVCOs con números de identificación mayores a 125 poseen la habilidad de utilizar el segundo canal del CAD para medir el voltaje de la batería u otras señales como parte del ciclo de prueba periódico. El intervalo del ciclo de prueba de estas unidades más recientes fue cambiado de 24 a 18 horas para que el tiempo de medición cambiara alrededor de 6 horas por día. El cambio en el tiempo de medición se realizó para ayudar a supervisar los sitios alimentados por energía solar que poseen un ciclo diario de carga y descarga. Otras señales podrían ser interconectadas para utilizar esta medición del CAD, pero el monitoreo del voltaje de la batería se ha escogido como la aplicación principal y es descrita a continuación.

El segundo canal de CAD se encuentra sólo disponible para el uso durante el ciclo de prueba, cuando el instrumento esté configurado para telemetría de un solo canal y el segundo canal del CAD no está conectado para medir señales sísmicas de baja ganancia. Vea la nota anterior, Utilización del Segundo Canal, para mayor información. Para habilitar el CAD del segundo canal durante el ciclo de prueba, los conectores en los pins 15 y 16 de U9 se encuentran seleccionados para una operación de un solo canal con el ciclo de prueba en funcionamiento.

Pin $15=5 \mathrm{~V}$ (ciclo de prueba en funcionamiento)

Pin $16=0 \mathrm{~V}$ (telemetría de un solo canal)

Es necesario construir un circuito de interconexión para adaptar el voltaje de la batería dentro del rango de 0 a 5 volts del CAD. El circuito puede ser un simple divisor de voltaje. En la Universidad del Instituto de Geofísica de Alaska construyeron el divisor basándose en un circuito tal que pudiera instalarse en el campo enchufándolo en el socket para U15. Vea la Figura $3 \boldsymbol{A}$. El circuito fue cableado de la siguiente manera:

Resistencia del $1 \%$ de $316 \mathrm{~K}$ entre los pins 6 y 8

Resistencia del $1 \%$ de $34.8 \mathrm{~K}$ entre los pins 4 y 6

Un puente del pin 8 al punto de soldadura $\mathbf{B}+$

Divisores sin aislamiento como éstos son cargados por el CAD, pero esto puede no ser importante mientras se mida el factor de escala. El Instituto de Geofísica informa que la linearidad es buena y un factor de escala de 80 cuentas por volt.

Si se necesitara un divisor aislado con buffer, el amplificador operacional en los pins 1, 2, y 3 de U3 puede ser utilizado como un amplificador inversor. Esto cancela la inversión en U15. Para lograr una ganancia unitaria en el CAD, R48 debe ser igual a R25. Un divisor de 5 a 1 ajusta el voltaje del panel solar descargado de más o menos 21 volts al rango del CAD. Conecte el divisor entre $\mathbf{B}+$ y tierra. El segundo potenciómetro de ajuste de nivel (P4) puede utilizarse para la calibración.

Los pulsos de medición del CAD son los últimos 12 del ciclo de prueba. El último pulso es el bit 
menos significativo. Los "unos" son los pulsos positivos (desviaciones positivas desde la frecuencia central). Los "ceros" son pulsos negativos (desviaciones negativas desde la frecuencia central). Existe un período de "descanso" en la frecuencia central entre cada pulso. Vea el dibujo en la nota anterior, Ciclo de Prueba del McVCO para tener un esquema de los 13 pulsos similares de ganancia y número de identificación. Cuando la conversión del CAD del segundo canal es habilitada, habrá un total de 25 de estos pulsos. Los pulsos pueden ser verificados iniciando un ciclo de prueba luego de un reset.

No espere que el voltaje de la batería del sistema de energía solar permanezca constante entre mediciones. Si la batería se está cargando y descargando, pérdidas internas se suman y restan del voltaje del circuito abierto de la batería. Los cambios de más de un volt pueden ocurrir cuando la batería pasa de carga a descarga. Cuando interprete las mediciones recuerde incluir cualquier caída de voltaje de los diodos entre la batería y el McVCO B+. 


\section{Utilización de la Salida de Potencia Conmutada}

El McVCO está diseñado para trabajar en lugares alejados alimentados por energía solar en donde el acceso es difícil o imposible durante ciertos períodos del año. El problema común de las estaciones en esas áreas es la energía intermitente. Por lo general, la sulfatación de la batería es la responsable y la energía sólo se puede obtener cuando el sol alcanza el panel solar. La estación deja de funcionar por la noche y vuelve a hacerlo por la mañana. El McVCO reacciona ante esta situación apagándose cuando el voltaje de la entrada es muy bajo, y poniéndose nuevamente en funcionamiento cuando el voltaje ha alcanzado niveles adecuados. Vea la descripción del circuito en la Parte II para más detalles. El apagado evita el ruido de descarga en el enlace de telemetría cuando los circuitos están bajos de energía, mientras que el encendido proporciona un reset limpio para el microcontrolador.

La carga más grande del sistema de energía no es el McVCO, sino el radiotransmisor. Algunos de los transmisores más nuevos requieren de 80 a $130 \mathrm{~mA}$, dependiendo del valor de la potencia de salida, mientras que el McVCO utiliza alrededor de $20 \mathrm{~mA}$. Apagar el transmisor cuando el McVCO está apagado tiene la ventaja de evitar una descarga adicional inútil y posiblemente perjudicial. También reduce el tiempo en que la estación no funciona. La salida de interrupción de la potencia del $\mathrm{McVCO}, \mathrm{SW}+$, tiene este fin.

Sin embargo, existen limitaciones para el uso de SW+ que deben considerarse antes de conectar una carga a la misma. El método de determinar la condición de la batería por medio del monitoreo del voltaje de entrada del McVCO tiene sus puntos débiles. Ante todo, el voltaje entre bornes no indica ni el estado de la carga de la batería ni la capacidad de suministrar energía, especialmente cuando la batería sufre sulfatación de placa. En segundo lugar, toda resistencia en las conexiones entre la batería y el instrumento, incluso la impedancia de la batería, produce una pérdida de voltaje de entrada del McVCO proporcional a la corriente (pérdida IR). Generalmente, esto no es un problema por el bajo consumo de corriente del instrumento, pero si se suministra una carga a través de $\mathrm{SW}+$, su corriente incrementa la pérdida IR. Esta pérdida IR aumentada puede causar oscilación si el encendido del SW+ provoca que el voltaje de entrada del McVCO caiga hasta el punto en que el instrumento se apague.

La secuencia de oscilación es la siguiente: el McVCO está APAGADO mientras el voltaje de la batería se recupera. Cuando el voltaje de la batería alcanza el umbral de encendido (11.5 V), el McVCO se ENCIENDE y se inicializa (reset). El McVCO enciende la carga de SW+. La corriente incrementada debido a la carga en $\mathrm{SW}+$ provoca una baja en el voltaje de entrada del McVCO por debajo del nivel crítico (10.5 V) y el McVCO se APAGA, APAGANDO SW+. El voltaje en la potencia de entrada aumenta porque ya no existe ninguna pérdida IR ni carga en la batería. El McVCO se ENCIENDE y se inicializa, etc.

Cuando la mayor parte de la pérdida de voltaje se deba a la resistencia de la línea, la oscilación puede ocurrir a una frecuencia bastante elevada y podría causar interferencia en el enlace de telemetría. Se han probado dos maneras de controlar la frecuencia de oscilación. Las primeras unidades tenían un atraso RC interconectado en el interruptor SW+ que mantenía baja la 
Parte III: Notas de Aplicación

frecuencia de oscilación. En unidades posteriores, el microcontrolador enciende SW+ después del ajuste automático a cero de 10 segundos al dar un reset. Este procedimiento detiene cualquier interferencia dirigida hacia el enlace de telemetría porque el instrumento está fijo a la frecuencia central durante el ajuste automático a cero. El reset es tan veloz que la unidad parecerá estar fija a esta frecuencia mientras el transmisor se enciende por un breve período.

Todo esto no significa que SW+ no deba ser usado nunca. El interruptor de carga puede utilizarse con éxito si se toman ciertas precauciones. La diferencia entre los puntos del interruptor de ENCENDIDO y APAGADO en la entrada de potencia del McVCO es de 1 volt. Si se permite un margen de seguridad de 0.5 volt, entonces con una carga total de $125 \mathrm{~mA}$ para el transmisor y el $\mathrm{McVCO}$, la resistencia total de la batería y las conexiones deben mantenerse a $4 \Omega,(0.5 \mathrm{~V} / 125$ $\mathrm{mA}$ ). El tamaño del cable no es ningún problema, a menos que exista un largo recorrido de cable entre el McVCO y la batería. La resistencia de un cable de calibre 22 es de $0.053 \Omega$ / metro. El problema está en la corrosión en las terminales de la batería, los conectores del cableado y tiras de conexiones. Se debe tener cuidado de prevenir la corrosión sellando estos puntos de conexión con soldadura, grasa o sellador para baterías.

Pilas de aire (pilas de aire de zinc y de aire alcalinas o Air Cells) se utilizan a veces como energía de respaldo para los sistemas que funcionan con energía solar. Estas tienen una resistencia interna bastante alta. Cuando las pilas de aire llegan al final de su vida útil, la combinación del voltaje reducido y la alta resistencia crea condiciones favorables para la oscilación de encendido del McVCO. Para ello se recomienda no utilizar el McVCO con sistemas que tengan sistemas de energía de respaldo con pilas de aire. El mejor lugar para colocar un interruptor de alimentación es en la batería y no en el VCO. Muchos nuevos reguladores de carga para celdas solares tienen interruptores de bajo voltaje (LVD, Low Voltage Disconnects) que interrumpen la alimentación a la batería y tienen menos posibilidades de oscilar que el interruptor del McVCO. 


\section{Conexión de Tres Componentes}

Las tarjetas del McVCO pueden conectarse a través de los conectores modulares J1 y J2 para permitir observaciones de eventos sísmicos de tres componentes. Tres McVCOs están conectados con la salida $\mathrm{J} 1$ de la primer tarjeta conectada a la entrada $\mathrm{J} 2$ de la segunda. J1 de la segunda tarjeta está conectada a $\mathrm{J} 2$ de la tercera. La salida de la tercer tarjeta tiene entonces las subportadoras para los tres componentes. Si las tarjetas operan en modo de ganancia dual, entonces se producen dos subportadoras de audiofrecuencia y un sitio de tres componentes puede producir hasta seis subportadoras. Es necesario ajustar las amplitudes de cada subportadora para que sean iguales en la salida de la última tarjeta de la cadena de McVCOs.

Cuando las tarjetas del McVCO se conectan a través de $\mathrm{J} 1$ y J2, la(s) subportadora(s) de una tarjeta se suma(n) a la(s) subportadoras de cualquiera de las tarjetas anteriores. Este subtotal se pasa y se suma a la(s) subportadora(s) siguiente(s) hasta que la última tarjeta de la cadena tiene todas las subportadoras presentes en la salida. Vea la Figura 8. El circuito de salida de cada etapa provoca una pérdida de amplitud de señal de manera que aquellas subportadoras que pasen por la mayor cantidad de etapas sean atenuadas lo más posible. Este esquema de atenuación irregular complica la ecualización.

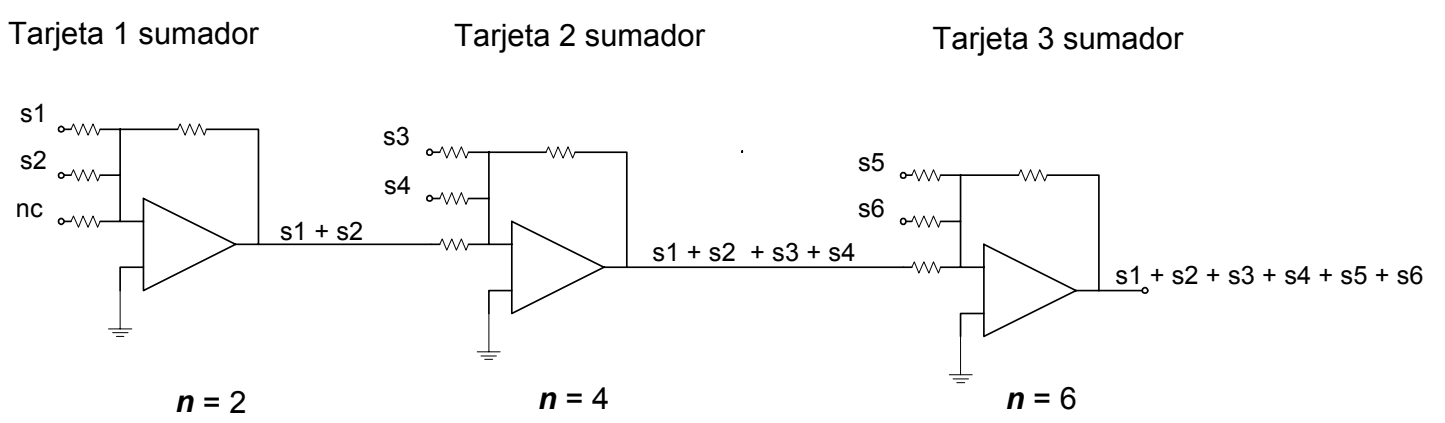

Figura 8. Los amplificadores sumadores de salida en cada tarjeta combinan las subportadoras de esa tarjeta con las subportadoras de tarjetas anteriores. Cuando s1=s $2=. . . s n$, el valor rms (la raíz cuadrada de los medios de los cuadrados) de la salida de cada tarjeta es proporcional a la raíz. cuadrada del número de subportadoras, $(n)^{1 / 2}$.

La ecualización puede comprobarse con un analizador de espectro o un a computadora personal equipada con harware y software para análisis espectral, pero es posible que estos instrumentos no estén disponibles. El método que sigue requiere un voltímetro rms de precisión para medir los niveles de la subportadoras cuando se realicen las calibraciones. El procedimiento puede ser ejecutado en el campo. 
Formas de onda complejas compuestas por ondas senoidales de igual amplitud, tienen una relación simple entre el voltaje rms y el voltaje rms que es común a cada parte. Si $\boldsymbol{n}$ es el número de las partes y $\boldsymbol{V}_{\mathbf{S}}$ es el voltaje rms común de cada parte, el voltaje rms total ( $\boldsymbol{V}_{\text {TOT }}$ ) de la forma de onda compleja será:

$$
V_{\text {TOT }}=\left(V_{\mathrm{S}}\right)(n)^{1 / 2}
$$

Para utilizar esta relación para ecualizar sitios con componentes múltiples, comience con la salida deseada, $\boldsymbol{V}_{\text {тот. }}$ Si $\boldsymbol{V}_{\text {тот }}$ no se conoce, obténgala de las especificaciones para el dispositivo, como puede ser un radiotransmisor, que la acepta como entrada, como un radiotransmisor. Si las especificaciones no están disponibles, intente adivinarla. $\boldsymbol{V}_{\text {тот }}$ puede reajustarse al final del procedimiento luego de que los componentes estén ecualizados. Un buen valor de prueba de $\boldsymbol{V}_{\text {TOT }}$ es $225 \mathrm{mV}$ rms.

Para nivelar las subportadoras a la salida de las tarjetas McVCO encadenadas, realice los siguientes ajustes en el orden indicado:

1. Si $\boldsymbol{n}$ es el número total de subportadoras en la salida de la última tarjeta de la cadena, ajuste cada subportadora en cada tarjeta a $\boldsymbol{V}_{\mathrm{S}}$, ya que $\boldsymbol{V}_{\mathrm{S}}=\boldsymbol{V}_{\text {TOT }} /(\boldsymbol{n})^{1 / 2}$. Mida la componente en TP5 y ajuste P5. Si la tarjeta está operando en modo de ganancia dual, mida la segunda subportadora en TP6 y ajuste P6.

2. Comenzando por la primer tarjeta de la cadena, mida el nivel rms de la señal de audio entre los puntos de prueba CAR+ y CAR- en el borde izquierdo de la tarjeta. Ajuste P7 para que la salida de la tarjeta sea $\left(V_{\mathrm{S}}\right)(\boldsymbol{n})^{1 / 2}$, donde $n$ es el número de todas las subportadoras en la salida de esa tarjeta. Vaya a CAR+ y CAR- de la tarjeta siguiente en la cadena y ajuste P7 en esa tarjeta para el nuevo valor de $\boldsymbol{n}$ como es requerido por el número de subportadoras en la salida de esa tarjeta. Vea la Figura 8 para tener un ejemplo de cómo cambia $\boldsymbol{n}$. Continúe hasta la última tarjeta.

3. P7 en la última tarjeta ajusta el nivel total de la forma de onda ecualizada. Mida entre CAR+ y CAR- mientras ajusta para obtener el valor conocido de $\boldsymbol{V}_{\mathrm{TOT}}$ o monitoree la respuesta del dispositivo que utiliza las subportadoras sumadas como su entrada. Si el valor inicial de $\boldsymbol{V}_{\mathrm{S}}$ está equivocado y el nivel de señal requerido está fuera del rango de ajuste de P7 en la última tarjeta, cambie $V_{\mathrm{S}}$ y repita el procedimiento.

Por ejemplo, se necesita una observación de ganancia alta y baja de un sitio sísmico de tres componentes. Tres tarjetas del McVCO que generan cada una dos subportadoras, se conectan a través de J1 y J2 para un total de seis subportadoras. La Figura 8 muestra cómo se combinan las subportadoras. La telemetría se realiza por un radiotransmisor de baja potencia. La desviación deseada del transmisor es $\pm 2.5 \mathrm{~Hz}$. Para ajustar las amplitudes de las subportadoras, debe hallarse el valor rms de la modulación que produce esta desviación. 
La sensibilidad del transmisor está fijada por el fabricante para una desviación de $5 \mathrm{Khz}$ en una modulación de $1.0 \mathrm{~V}$ rms. La desviación es proporcional al voltaje máximo y no al voltaje rms. El fabricante utiliza un tono único para especificar las características de modulación. En consecuencia, la desviación máxima es $5 \mathrm{KHz} /(2)^{1 / 2}(1.0 \mathrm{~V} \mathrm{rms})=3.54 \mathrm{KHz} / \mathrm{V}$. Al usar la sensibilidad máxima de desviación, el voltaje de modulación máximo para una desviación de 2.5 $\mathrm{KHz}$ es $(2.5 / 3.54) \mathrm{V}$ pico $=0.71 \mathrm{~V}$ pico.

Para ecualizar las subportadoras, se necesita el valor eficaz del voltaje de modulación.

Desafortunadamente, no existe una manera simple de convertir una forma de onda compleja de valor pico a valor rms. Sin embargo, puede realizarse una aproximación. La Tabla 1 muestra el factor intermedio de cresta para las formas de onda ecualizadas compuestas por frecuencias de telemetría $(680,1020, \ldots 3060)$. El factor de cresta es el cociente del voltaje de pico entre el voltaje rms. En la Tabla 1, una forma de onda de seis componentes tiene un factor medio de cresta de 2.21. Por lo tanto, el valor eficaz aproximado del voltaje de modulación es: máximo $0.71 \mathrm{~V}$ pico / $2.21=321 \mathrm{mV}$ rms.

\begin{tabular}{|l|l|l|l|l|l|l|l|l|}
\hline $\begin{array}{l}\text { Número de } \\
\text { componentes }\end{array}$ & 1 & 2 & 3 & 4 & 5 & 6 & 7 & 8 \\
\hline $\begin{array}{l}\text { Factor Medio de } \\
\text { Cresta }\end{array}$ & $(2)^{1 / 2}$ & 1.92 & 2.12 & 2.18 & 2.20 & 2.21 & 2.22 & 2.23 \\
\hline
\end{tabular}

Tabla 1. El factor medio de cresta de las formas de onda complejas de telemetría con diferentes números de componentes. Los componentes tienen amplitudes iguales y sus frecuencias son las frecuencias de telemetría sísmica $(680,1020, . .3060)$. El factor de cresta es el cociente, $V$ pico/V rms. El cálculo del factor de cresta se realizó sumando todas las combinaciones posibles de frecuencias de telemetría en fases aleatorias durante muchas pruebas para poder hallar el voltaje medio de pico a pico para cada forma de onda con más de un componente.

El valor de cada subportadora $\left(\boldsymbol{V}_{\mathrm{S}}\right)$ es entonces $\boldsymbol{V}_{\mathrm{S}}=(321) /(6)^{1 / 2}=131 \mathrm{mV} \mathrm{rms}$. El paso $1 \mathrm{del}$ procedimiento se completa ajustando cada subportadora de cada tarjeta a $131 \mathrm{mV}$ rms en TP5 o TP6 respectivamente con P5 y P6.

La Tarjeta 1 tiene dos subportadoras en su salida. P7 en la Tarjeta 1 debe ajustarse para que entre los puntos de prueba CAR+ y CAR- la salida sea de $131(2)^{1 / 2} \mathrm{mV} \mathrm{rms}=185 \mathrm{mV} \mathrm{rms}$.

La Tarjeta 2 tiene cuatro subportadoras en su salida. P7 en la Tarjeta 2 debe ajustarse para que entre los puntos de prueba CAR+ y CAR- la salida sea de $131(4)^{1 / 2} \mathrm{mV} \mathrm{rms}=262 \mathrm{mV} \mathrm{rms}$.

La Tarjeta 3 tiene seis subportadoras en su salida. P7 en la Tarjeta 3 debe ajustarse para que entre los puntos de prueba CAR+ y CAR- la salida sea de 131 ( 6) ${ }^{1 / 2} \mathrm{mV}=321 \mathrm{mV} \mathrm{rms}$.

Esto completa la calibración de ejemplo. La forma de onda compensada de $321 \mathrm{mV}$ rms producirá un promedio de $\pm 2.5 \mathrm{KHz}$ de desviación del transmisor. 